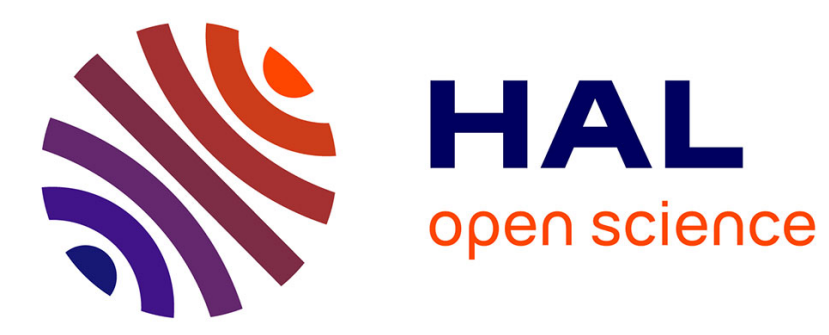

\title{
Multi-electron coincidence spectroscopy: double photoionization from molecular inner-shell orbitals
}

Pascal Lablanquie, F Penent, Y Hikosaka

\section{To cite this version:}

Pascal Lablanquie, F Penent, Y Hikosaka. Multi-electron coincidence spectroscopy: double photoionization from molecular inner-shell orbitals. Journal of Physics B: Atomic, Molecular and Optical Physics, 2016, 49 (18), pp.182002 10.1088/0953-4075/49/18/182002 . hal-01387100

\section{HAL Id: hal-01387100 https://hal.sorbonne-universite.fr/hal-01387100}

Submitted on 25 Oct 2016

HAL is a multi-disciplinary open access archive for the deposit and dissemination of scientific research documents, whether they are published or not. The documents may come from teaching and research institutions in France or abroad, or from public or private research centers.
L'archive ouverte pluridisciplinaire HAL, est destinée au dépôt et à la diffusion de documents scientifiques de niveau recherche, publiés ou non, émanant des établissements d'enseignement et de recherche français ou étrangers, des laboratoires publics ou privés. 


\title{
Multi-electron coincidence spectroscopy: double photoionization from molecular inner-shell orbitals
}

\author{
P. Lablanquie ${ }^{1}$, F. Penent ${ }^{1}$ and Y. Hikosaka ${ }^{2}$ \\ ${ }^{1}$ Sorbonne Universités, UPMC Univ Paris 06, CNRS, LCP-MR (UMR 7614), \\ 11 rue Pierre et Marie Curie, 75231 Paris Cedex 05, France \\ ${ }^{2}$ Graduate School of Medicine and Pharmaceutical Sciences, University of Toyama, 930-0194, Japan
}

\begin{abstract}
The interest of molecular double core holes was predicted in 1986 by Cederbaum et al who showed that their spectroscopy can be more informative than that of the single core holes, especially when the holes are located at different sites in the molecule (Cederbaum et al $J$. Chem. Phys. 85 (1986) 6513). Their experimental study in single photon formation had to await 2009-2010 with progress in synchrotron sources and development of efficient multi-electron coincidence experiments based on a magnetic bottle time of flight spectrometer. At the same time the advent of X-rays Free Electron Lasers opened the possibilty to create them in a two-photon process, and motivated new theoretical studies of their properties. We will illustrate here with a few examples, the progress done recently in the field, including formation of double core holes by double core photoionization, their spectroscopy and decay paths, and the related process of simultaneous core ionization and core excitation.
\end{abstract}

\section{Introduction}

Single photon double ionization from valence shell orbitals has been extensively studied as a probe of electron correlation. Especially the simplest atomic $\mathrm{He}$ and molecular $\mathrm{H}_{2}$ targets have attracted much experimental and theoretical work [1] [2] [3]. It is only in the last five years that it became possible to detect double photoionization in the deeper inner-shell molecular orbitals. Of primary interest is the double ionization in the K-shell, as it cannot be accessed by cascade Auger decay from a deeper shell. Two possibilities arise in molecules: either the two electrons are ejected from the same $\mathrm{K}$ shell of a given atom in the molecule (1-site Double Core Holes (DCH) or $\mathrm{K}^{-2}$ ) or they originate from two different K-shells, that is from two different atoms in the molecule (2-site $\mathrm{DCH}$ or $\left.\mathrm{K}^{-1} \mathrm{~K}^{-1}\right)$. It is the multi-electron coincidence technique, based on the very efficient magnetic bottle time-of flight apparatus, which made possible the experimental observation of these weak cross section processes. $\mathrm{K}^{-2}$ double ionization is typically $10^{-3}$ compared to the single ionization $\mathrm{K}^{-1}$, while the weaker $\mathrm{K}^{-1} \mathrm{~K}^{-1}$ double ionization drops to a typical $10^{-5}$ value. The first interest of these DCH states is 
their spectroscopy, which was predicted to be more sensitive to the chemical environment than the $\mathrm{K}^{-1}$ single core-hole spectroscopy. The demonstration was established by the experimental study of the $\mathrm{C}_{2} \mathrm{H}_{2 n}$ series $(\mathrm{n}=1,2,3)$, testing the predictions made by Cederbaum et al in 1986 [4] that $\mathrm{K}^{-1} \mathrm{~K}^{-1}$ spectroscopy does differentiate these species. Great efforts have been devoted to the calculation of DCHs energies, using different theoretical approaches and showing that they give access to the relaxation energy upon $\mathrm{K}$ shell ionization. In parallel the advent of intense x-rays Free Electron Lasers (XFELs) made it possible to observe also DCHs, but by populating them not in a single photon double ionization process, but in sequential two photon ionization, each photon extracting one $\mathrm{K}$ shell electron from the molecule. The key point is the duration of the XFEL laser pulse which needs to be of the order of the lifetime of the intermediate $\mathrm{K}^{-1}$ state (typically in the fs range). DCHs have been indeed probed with XFELs, however a careful analysis is needed due to the superposition of many other multiphoton processes. Another obstacle is the difficulty to take advantage of coincidence techniques in the present XFELs experiments, which are only partly compensated by the newly developed covariance techniques. The coincidence technique is, on the opposite, perfectly well suited to study single photon double ionization with synchrotron radiation. It allows for instance the observation of the decay of each DCH state by the successive emission of two Auger electrons, giving access to the intermediate and final states. Comparison with calculations showed the presence of nuclear motion during the Auger decay. The most recent development is the study of the closely related process in which a single photon simultaneously ionizes a $\mathrm{K}$-shell electron and excites another one (noted $\mathrm{K}^{-2} \mathrm{~V}$ ). Theoretical modeling showed it offers rich spectroscopic information due to the superposition with equal probabilities, of the direct path and the conjugate one, which obey different selection rules.

In this review we will summarize the progress recently achieved in these different subjects, and extend the previous reviews that have already been published on the subject [5] [6] [7] [8] [9].

\section{Experimental observation of single photon double ionization from molecular inner-shell orbitals}

Single photon double core ionization being a very weak process, its detection had to await the development of a very efficient experimental technique: multi-electron coincidence spectroscopy using a magnetic bottle spectrometer. Such a spectrometer is based on the original design of Kruit and Read [10] and was first implemented to study multiple ionization by J. Eland [11]. It has been used during the last 13 years to study a variety of multi-photoionization processes. The merit is that electrons emitted over $4 \pi$ solid angle are collected and detected with a high probability (up to $70 \%$, depending on the kinetic energy of the electron), so that 
efficient coincidence detection of all emitted electrons is possible; moreover the long time-of-flight tube (over $2 \mathrm{~m}$ ) ensures a good kinetic energy resolution (of 1-2\% of the kinetic energy). In the core double photoionization case considered here, the double core hole states produced after ejection of the two photoelectrons are unstable and decay mainly by successive emission of two Auger electrons. As will be explained below, it is the coincidence detection of at least 3 of the released electrons that has enabled one to isolate these weak double core ionization processes from the dominant single core ionization ones.

\section{1 $\mathrm{K}^{-2}$ states: 1-site Double Core Holes or Hollow molecules}

In this case, the two $\mathrm{K}$ shell electrons from a given atom $\mathrm{A}$ of a molecule are ejected following the absorption of a single photon, leading to what is called a 'hollow molecule'. The complete reaction for the creation and the subsequent decay of the 1-site double core hole, can be written in the following way:

$$
\begin{aligned}
& \mathrm{hv}+\mathrm{AB} \rightarrow \quad \mathrm{AB}^{2+}\left(\mathrm{K}^{-2}\right)+2 \mathrm{e}^{-} \text {Photoelectron } \\
& \rightarrow \mathrm{AB}^{3+}\left(\mathrm{K}^{-1} \mathrm{v}^{-2}\right)+\mathrm{e}^{-} \text {Auger Hypersatellite } \\
& \rightarrow \mathrm{AB}^{4+}\left(\mathrm{v}^{-4}\right)+\mathrm{e}^{-} \text {2nd step Auger }
\end{aligned}
$$

where $\mathrm{K}$ is the $\mathrm{K}$-shell orbital of the atom $\mathrm{A}$, and $\mathrm{v}$ is a molecular valence orbital.

The first emitted Auger electron is called 'hypersatellite' and is faster than the ' 2 nd step Auger' electron emitted afterwards (1c). We will illustrate this process with our first observation of 1-site double core photoionization. It was performed in $\mathrm{N}_{2}$ at Photon Factory (Japan) using photons of $h v=1110 \mathrm{eV}$ [12]. Fig. 1 shows the experimental evidence; it relies on the coincident detection of the four electrons emitted in reaction (1) and on different energy representations of this 4-dimension problem. Fig. 1(a) shows the energy correlation between two photoelectrons when detected in coincidence with the two Auger electrons chosen in the kinetic energy ranges of 375-450 eV and 300-375 eV. Diagonal lines at a constant sum of the two photoelectrons' energies are observed and correspond to the formation of different $\mathrm{K}^{-2}$ states. The binding energy of these states is deduced from the equation for energy conservation: (Binding Energy of $\mathrm{K}^{-2}$ states $)=\mathrm{h} v-\left(\mathrm{KE}_{\mathrm{e} 1}+\mathrm{KE}_{\mathrm{e} 2}\right)$, and is represented in Fig. 1(b). In addition to the first peak at $902.55 \mathrm{eV}$ which corresponds to the $\mathrm{K}^{-2}$ ground state, satellite peaks appear at higher binding energy; they result from the additional excitation of a valence electron to an unoccupied orbital. It is interesting to note that the relative contribution of $\mathrm{K}^{-2}$ satellites is higher $(\sim 25 \%)$ than that of the satellites in $\mathrm{K}^{-1}$ single ionization $(\sim 10 \%)$ due to the stronger perturbation of the valence electrons introduced by the removal of two K-shell electrons instead of one. Finally, the complete Auger electron spectrum associated with the decay of the $\mathrm{K}^{-2}$ 
ground state is shown in Fig. 1c. It is seen that the energy regions of the hypersatellite Auger [375-430 eV] and of the second-step Auger electron [300-375 eV] are clearly separated. Energy correlation between these two Auger electrons can also be represented, and give information on the intermediate $\mathrm{N}_{2}{ }^{3+}$ and final $\mathrm{N}_{2}{ }^{4+}$ states with a limited resolution due to the $\sim 6 \mathrm{eV}$ experimental resolution for fast Auger electrons [12].

Information on the double photoionization process can be retrieved from experiment. For instance the intensity distribution along the diagonal lines in Fig.1 (a) demonstrates a continuous energy sharing between the two photoelectrons, which indicates a dominant direct double ionization process. It can be compared to the He double ionization at a lower excess energy of $\sim 13 \mathrm{eV}$ instead of $208 \mathrm{eV}$ here, if we consider the $\mathrm{Z}^{2}$ scaling effect due to the effective charge $\mathrm{Z}$ of the core. The flatness of both distributions in He [13] and $\mathrm{N}_{2}$ suggests a dominant contribution at these low excess energies of the knock-out process resulting from the internal collision of a first $1 \mathrm{~s}$ electron on the other 1s electron of the same atom [12]. Another experimental information is the intensity of the core double photoionization process: the ratio of double to single photoionization can be directly extracted from the coincidence data set, by comparing the number of detected events and taking into account detection efficiencies; they are typically of the order of $10^{-3}$, and seem to decrease with the nuclear charge Z [14]. They are of similar order of magnitude as for the $\mathrm{K}$-shell double ionization of light atoms $(12 \leq \mathrm{Z} \leq 24)$ that have been extensively studied by their radiative $\mathrm{K} \alpha^{\mathrm{h}}$ hypersatellite $\mathrm{X}$-ray emission [15]. It would be interesting to explore the photon energy and element dependence of these double to single core photoionization ratios, in the same manner as it was done for light atoms. However this is not an easy experimental task, because of the coincidence technique and overlap of photoelectron and Auger energies when excess energy becomes large.

Fig. 2 shows another example of the first observations of $\mathrm{K}^{-2}$ states with synchrotron, obtained in $\mathrm{CH}_{4}$ and $\mathrm{NH}_{3}$ by the group of R. Feifel [16]. In this case the signal was retrieved from the analysis of triple coincidence events involving detection of the two photoelectron and the fastest hypersatellite Auger electron. The lower statistics of the data in Fig 2 compared to Fig 1 can be assigned to the lower accumulation time ( $2 \mathrm{~h}$ versus $12 \mathrm{~h}$ ), while the higher background originates probably from the less stringent conditions in signal filtering.

Since these two pioneer experiments, 1-site DCHs have been observed in a variety of molecules. Table 1 lists the measured binding energies of $\mathrm{K}^{-2}$ ground states that have been reported in the literature up to now. It is seen that much remains to be done! Satellite states have been clearly observed in some cases and successfully calculated. This is the case for the $\mathrm{N}_{2}$ and $\mathrm{CO}\left(\mathrm{O} \mathrm{K}^{-2}\right)$ satellite states calculated by Tashiro et al [17]. The $\mathrm{N}_{2}$ result is displayed in Fig. 3 and the satellite states of the isoelectronic $\mathrm{C}_{2} \mathrm{H}_{2}$ molecule, calculated by Lablanquie et al [18] is shown in Fig. 4 (b). It is seen that a good understanding of the origin of the satellite states is achieved (the main satellite peak is ascribed to a $\pi-\pi$ excitation, see original 
publications for assignments of the other components) and that their intensities are fairly well reproduced.

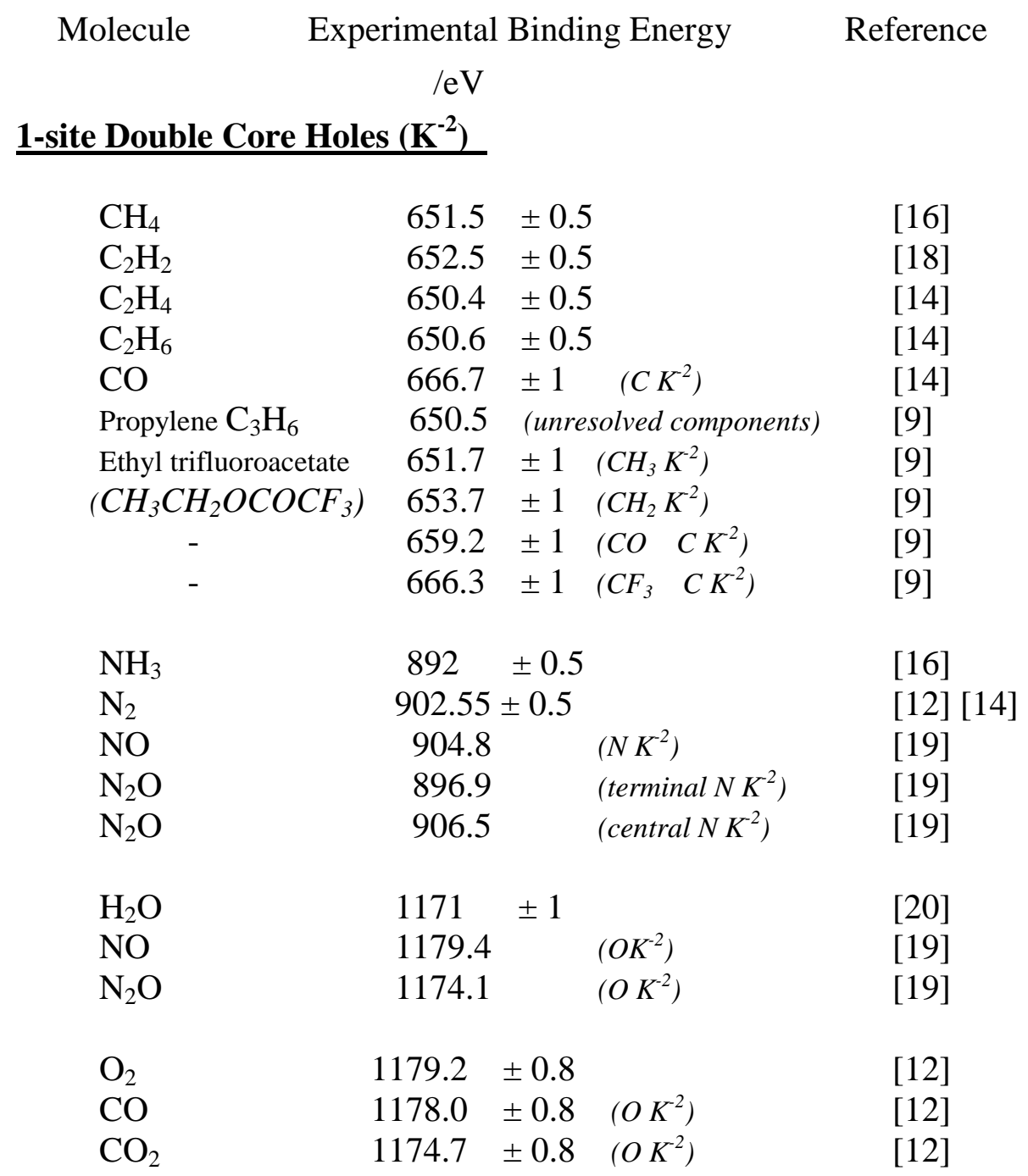

\begin{tabular}{lccc} 
2-site Double Core Holes $\left(\mathbf{K}^{\mathbf{- 1}} \mathbf{K}^{\mathbf{- 1}}\right)$ & \\
\hline $\mathrm{C}_{2} \mathrm{H}_{2}$ & 595.6 & \pm 0.5 & {$[18]$} \\
& 596.0 & \pm 0.5 & {$[14]$} \\
$\mathrm{C}_{2} \mathrm{H}_{4}$ & 593.3 & \pm 0.5 & {$[14]$} \\
$\mathrm{C}_{2} \mathrm{H}_{6}$ & 590.2 & \pm 0.5 & {$[14]$} \\
Propylene $\mathrm{C}_{3} \mathrm{H}_{6}$ & 591 & & {$[9]$} \\
& & & \\
$\mathrm{CO}$ & 855.4 & \pm 1 & {$[14]$} \\
$\mathrm{N}_{2}$ & 835.9 & \pm 1 & {$[14]$}
\end{tabular}

TABLE I : Experimental binding energies of DCH states, as obtained from single photon 
double ionization experiments

\section{2 $\mathrm{K}^{-1} \mathrm{~K}^{-1}$ states: 2-site Double Core Holes}

Here, two K-shell electrons from different atoms in a molecule are ejected following the absorption of a single photon. The creation and the subsequent decay of the 2-site double core hole, can be written in the following way:

$$
\begin{aligned}
\mathrm{h} v+\mathrm{AB} \rightarrow \quad\left[\mathrm{A}\left(\mathrm{K}^{-1}\right)\right. & \left.\mathrm{B}\left(\mathrm{K}^{-1}\right)\right]^{2+}+2 \mathrm{e}^{-} \text {Photoelectron } \\
& \rightarrow \quad\left[\mathrm{A} \mathrm{B}\left(\mathrm{K}^{-1}\right) \mathrm{v}^{-2}\right]^{3+} \text { or }\left[\mathrm{A}\left(\mathrm{K}^{-1}\right) \mathrm{B} \mathrm{v}^{-2}\right]^{3+}+\mathrm{e}^{-} \text {1st step Auger } \\
& \rightarrow \mathrm{AB}^{4+}\left(\mathrm{v}^{-4}\right)+\mathrm{e}^{-} \text {2nd step Auger }
\end{aligned}
$$

Note that the successive decay of the K-shell holes means that it is either of the two sites which decays first.

As this double photoionization process relies on the weak electron correlation between two K-shell electrons localized at different sites in the molecule, its intensity was expected to be extremely low, making its observation possibly below experimental possibilities. It turned to be of the order of $10^{-5}$ compared to single photoionization, which is at the limit of detection. The first experimental observation of single photon 2-site K-shell double ionization was obtained on the $\mathrm{C}_{2} \mathrm{H}_{2}$ molecule [18] and is presented in Fig. 4. Four-fold coincidence between the two photoelectrons and the two Auger electrons, which are respectively chosen in the 270$320 \mathrm{eV}$ (Auger hypersatellite) and $200-270 \mathrm{eV}\left(2^{\text {nd }}\right.$ step Auger) ranges reveals in Fig 4 (a) the main $\mathrm{K}^{-2}$ line at $652.5 \pm 0.5 \mathrm{eV}$ and satellite states at the higher binding range, in the same way as presented above for the $\mathrm{N}_{2}$ isoelectronic molecule. One difficulty to detect the $\mathrm{K}^{-1} \mathrm{~K}^{-1}$ signal in the present case (where atoms A and B are both Carbon atoms) is that the two Auger electrons emitted in the decay (Eq. 2) lie in the same energy range of 230-250eV and could not be both detected because of the $\sim 15 \mathrm{~ns}$ dead time of the detector. It was however possible to isolate the characteristic $\mathrm{K}^{-1} \mathrm{~K}^{-1}$ peak in three-fold coincidences between the two photoelectrons and one of the two subsequent Auger electrons. The peak appears in Fig 4 c) at a binding energy of $595.6 \pm 0.5 \mathrm{eV}$, very close to the calculated value of $595.86 \mathrm{eV}$ [18].

An important experimental achievement was to demonstrate the validity of the original prediction form Cederbaum et al [4] that chemical shifts of DCHs, and especially of the $\mathrm{K}^{-1} \mathrm{~K}^{-1}$ ones, are more informative than those of single core holes. He used the $\mathrm{C}_{2} \mathrm{H}_{2 n}(\mathrm{n}=1,2,3)$ series and predicted that these three molecules could be differentiated by the energy of their $\mathrm{K}^{-1} \mathrm{~K}^{-1}$ ground states, whereas the determination of the $\mathrm{K}^{-1}$ or even the $\mathrm{K}^{-2}$ ground state binding energy is not sufficient. The experimental confirmation was done in 2013 [14] and is illustrated in Fig. 5. Three-fold coincidences between two photoelectrons and one of the two Auger electron emitted in reaction (2) reveal the $\mathrm{K}^{-1} \mathrm{~K}^{-1}$ signal, as explained previously. Note that the $\mathrm{K}^{-2}$ states 
also appear in the spectra, because the energy range for the $\mathrm{K}^{-1} \mathrm{~K}^{-1}$ Auger electrons corresponds also to that of the second step Auger electron emitted during the decay of the $\mathrm{K}^{-2}$ state (See reaction 1). The experimental result fully confirms the predictions of Cederbaum et al: the three molecules are differentiated by their $\mathrm{K}^{-1} \mathrm{~K}^{-1}$ binding energy, whereas the $\mathrm{K}^{-2}$ ones are similar for $\mathrm{C}_{2} \mathrm{H}_{4}$ and $\mathrm{C}_{2} \mathrm{H}_{6}$. The sensitivity of the $\mathrm{K}^{-1} \mathrm{~K}^{-1}$ binding energy comes from the fact that it depends on the $\mathrm{C}-\mathrm{C}$ bond length, through the repulsion energy between the two core holes (see $\$ 5.1$ ).

Since then, 2-site double core ionization has been detected in a few other molecules, as reported in Table I, but results are still few, as the weakness of the signal makes their detection very difficult. Note that $\mathrm{K}^{-1} \mathrm{~K}^{-1}$ states have also been detected in four-fold coincidence experiments. This is either because the two K-shell holes are on atoms of different nature, implying different energy ranges for the 2 emitted Auger electrons in reaction (2), such as in $\mathrm{CO}$ [14], or because the dead time of the detector was reduced. A value of 6ns, corresponding to a to a dead zone in energy of $\sim 12 \mathrm{eV}$ for electrons of $240 \mathrm{eV}$, made it possible to observe $\mathrm{K}^{-1} \mathrm{~K}^{-1}$ signals in $\mathrm{C}_{2} \mathrm{H}_{4}$ and propylene molecules [9]. A subsequent advantage is that the background is much reduced in 4-fold coincidences.

An estimate of the probability for the two-site core double photoionization in $\mathrm{C}_{2} \mathrm{H}_{2}$ molecules was presented in [18]. Although overestimated by a factor 3 , the predicted values suggest that the mechanism is a knock-out process in which the first (primary) K-shell electron absorbs a photon and then ejects by internal collision a K-shell electron from the neighboring atom. The model predicts a $1 / \mathrm{R}^{2}$ dependence of the $\mathrm{K}^{-1} \mathrm{~K}^{-1}$ cross-section on the $\mathrm{C}-\mathrm{C}$ bond length. Such a dependence was experimentally observed in the series of molecules $C_{2} H_{2 n}(n=1$, 2, 3) [14], which brings a further argument for the validity of the knock-out picture.

\section{Auger decay of Double Core Holes}

The great advantage of the coincidence experiments, is that they enable us to isolate efficiently the Auger spectra associated with the decay of well-defined double core hole states.

Fig. 6 illustrates this with the Auger decays of double core hole states in $\mathrm{C}_{2} \mathrm{H}_{2}$. Decay of the $\mathrm{K}^{-2} \mathrm{DCH}$ main line (b) and of the main satellite line (c) present similar characteristics as what is observed for the isoelectronic $\mathrm{N}_{2}$ case in Fig 1 (c): the first hypersatellite Auger electron is found in the 270-340 eV kinetic energy range (solid line) and is faster than K-VV Auger electrons in Fig 6 (d). The second step Auger electron released in the last step of the decay is found in the 200-270 eV range (dotted lines), at an energy similar but slightly lower than the K-VV Auger. The predominance of the spectator decay for the DCH satellite state is evidenced by the similarity of the Auger spectra in Fig. 6(b) and 6(c). A shoulder at $314 \mathrm{eV}$ in 
Fig. 6(c) indicates nevertheless the presence of the participator decay. Concerning the $\mathrm{K}^{-1} \mathrm{~K}^{-1}$ DCH decay, Fig. 6 (a) demonstrates that the two Auger electrons are emitted with similar energies and in the 220-250 eV range. The binding energies of the $\mathrm{C}_{2} \mathrm{H}_{2}{ }^{3+}$ intermediate states created in the first step of the Auger cascade are indicated on the top of Fig. 6. They are obtained by considering energy conservation during the 2 first steps of equations (1) and (2). Fig 6 a) and b) suggest that similar $\mathrm{C}_{2} \mathrm{H}_{2}{ }^{3+}$ intermediate states are reached in the decay of $\mathrm{K}^{-1} \mathrm{~K}^{-1}$ and $\mathrm{K}^{-2} \mathrm{DCHs}$, whereas more excited intermediate states result from the decay of $\mathrm{K}^{-2}$ satellite DCHs, as shown in Fig 6 c).

Fig. 7 addresses the Auger decays of double core hole states in CO [14]. The interest is the comparison of the experiment with calculations, which bring clues to the decay mechanism of the $\mathrm{K}^{-1} \mathrm{~K}^{-1} \mathrm{DCH}$. Calculations use the model detailed in Ref. [21]. Fig 7b shows that the decay of the 1-site $\mathrm{C}\left(\mathrm{K}^{-2}\right) \mathrm{DCH}$ (Fig $\left.7 \mathrm{~b}\right)$ is quite well reproduced; it displays the contributions of the hypersatellite Auger electron in the $250-320 \mathrm{eV}$ range, and of the second step Auger electron below $250 \mathrm{eV}$. As for the Auger decay of the $\mathrm{K}^{-1} \mathrm{~K}^{-1} \mathrm{DCH}$ (Fig 7c), the model assumes non concerted successive Auger decay on the two sites. In this case two pathways are possible where the first Auger occurs on the $\mathrm{O}$ site and the second on the $\mathrm{C}$ site, or the opposite. The calculations predicts close ratio for these two pathways because of the similar lifetimes of the $\mathrm{C}$ and $\mathrm{O}$ single core holes. The theoretical Auger spectra for the two decay pathways are plotted in Fig. 7 c). The total Auger spectrum is the sum of the two channels, and agrees well with experiment. It confirms that the Auger electrons emitted from the $\mathrm{C}$ site upon decay of the 2-site $\mathrm{K}^{-1} \mathrm{~K}^{-1} \mathrm{DCH}$ lie in the same $200-250 \mathrm{eV}$ energy range, slightly slower than the Auger electrons of the $\mathrm{C}$ single core holes, but similar to the second step Auger electron of the $\mathrm{C} \mathrm{K}^{-2} \mathrm{DCH}$. It is interesting to note that calculation predicts a slightly faster $\mathrm{C}$ Auger electron for the decay of the $\mathrm{K}^{-1} \mathrm{~K}^{-1}$ state, if it is the $\mathrm{C}$ site that decays first rather than the $\mathrm{O}$ one.

The next step in the description of the Auger decay of DCHs is to consider the effect of Coulomb explosion during the successive Auger decays. In spite of the low experimental resolution of the coincidence technique, comparison with calculations made it possible to demonstrate in the isoelectronic molecules $\mathrm{C}_{2} \mathrm{H}_{2}$ and $\mathrm{N}_{2}$, the effect of the nuclear motion once the first Auger electron has been emitted [22]. This is illustrated in Fig. 8 and 9. Fig. 8 compares the experimental (a) and theoretical (b) two-dimensional Auger spectrum associated with the decay of the $\mathrm{C}_{2} \mathrm{H}_{2} \mathrm{~K}^{-2}$ ground state. It confirms the assignment of the hypersatellite and second step Auger electrons discussed in Fig.6. The overall agreement between calculation and experiment is good, but a closer look shows that the second step Auger electron is less structured than predicted. This is better seen in Fig. 9 a), where 1-dimensional complete Auger 
spectra, are presented for the $\mathrm{N}_{2}$ molecule. They are obtained by the projection of 2-dimensional Auger spectra such as those in Fig. 8. The results are similar in $\mathrm{C}_{2} \mathrm{H}_{2}$ and $\mathrm{N}_{2}$, but nuclear dynamics calculations can be done only for the simpler $\mathrm{N}_{2}$ diatomic case

Fig 9 (a), shows that the calculation, done without the effect of nuclear motion, agree with experiment for the shape of the hypersatellite Auger, but predicts two well separated peaks in the second step Auger spectrum, which are broader and less separated in the experiment. Inclusion of the nuclear motion in the calculation, that is nuclear dynamics during the lifetime of the $\mathrm{K}^{-2}$ initial and $\mathrm{K}^{-1} \mathrm{v}^{-2}$ intermediate states (see Equation 1) leads to the spectra in Fig 9 (b). The shapes of the spectra in the 1st Auger component are very similar to each other, which suggests that the effect of nuclear motion may be small in the 1st Auger decay. In contrast, the effect of nuclear motion is significant in the 2nd Auger. This effect can be understood if we consider the potential energy curves of the initial $\mathrm{K}^{-2}$ state which is bound, and of the $\mathrm{K}^{-1} \mathrm{v}^{-2}$ intermediate states and $\mathrm{v}^{-4}$ final states which are all dissociative, with a steeper gradient for the final than the intermediate states [22]. Note that extraction of the two K-shell electrons from the same site in the molecule is in principle not expected to affect the bounding properties of the molecule, and the intermediate $\mathrm{K}^{-2}$ state should be bound, similar to the neutral ground state or to the single core $\mathrm{K}^{-1}$ one. This is in agreement with calculations for $\mathrm{C}_{2} \mathrm{H}_{2}$ and $\mathrm{N}_{2}$ [22]; but Inhester et al [23] predicted that it is not the case for $\mathrm{H}_{2} \mathrm{O}$, where the $\mathrm{K}^{-2}$ state is found to be dissociative, opening new nuclear dynamics possibilities.

\section{Double Core Holes as observed in two photon processes at XFELs}

The advent of the new XFELs sources opened new perspectives with their unprecedented brightness, which can be up to 10 orders of magnitude higher than conventional synchrotron sources. One of their potential applications was predicted to be the study of DCHs, thanks to their sequential formation in a two photon process. Calculations preceded experiments and anticipated the power of this new spectroscopic tool, ' $\mathrm{x}$-ray two-photon photoelectron spectroscopy', with the example of the para-aminophenol molecule [24]. The first gas phase experiments with the first XFEL source, the LCLS (Linac Coherent Light Source), revealed indeed the formation of DCHs in neon atoms [25]. The first DCHs in molecules were observed shortly later in $\mathrm{N}_{2}$ [26] [27]. Both Auger and photoelectron spectra associated to DCHs formation were observed in [27] while the molecular frame angular distribution of the hypersatellite Auger electron from aligned $\mathrm{N}_{2}$ molecules was obtained in [26].

Fig. 10 shows the first evidence of DCHs formation with XFELs in a photoelectron spectrum [27]. On the low energy side of the $\mathrm{K}^{-1}$ photoelectron corresponding to the single 
ionization of the $\mathrm{N}_{2}$ molecule, a clear peak is observed at the expected position of the single site DCH photoelectron peak. However, this energy is also close to the calculated positions of peaks associated with core ionization of a triply valence-ionized $\mathrm{N}$ atom, and these processes cannot be distinguished in the photoelectron data. This illustrates the complexity of the processes occurring with XFEL excitation: sequential absorption of several $\mathrm{x}$-ray photons occurs and the molecule has the possibility to Auger decay and dissociate in the course of this photon absorption. This makes the interpretation of XFELs spectra difficult and dependent on theoretical modelization. A substantial progress was achieved when the XFEL pulse duration could be shortened below the core-hole lifetime. In this way, one can optimize the probability of two photon absorption before the first created hole can decay. In short, one "beats" the Auger decay clock. This was demonstrated by Berrah et al in CO [28] and used later to investigate a series of molecules including $\mathrm{N}_{2}$ [29]. Fig. 11 illustrates the method and demonstrates the progress compared with Fig 10. The XFEL pulse duration has been decreased from the previous $280 \mathrm{fs}$ to $10 \mathrm{fs}$, that is of the same order of magnitude as the N1s lifetime (6.4 fs). Another important parameter is the intensity of the XFEL radiation at the interaction zone which is further increased by tightly focusing the laser beam. Comparison of the focused (blue) and unfocussed (green) spectra allows one to extract the nonlinear contributions to the photoelectron signal and to observe clearly both single-site and two-site DCHs. The spurious peaks that complicated the photoelectron spectrum in Fig. 10 are now reduced to a single peak 'PAP' which corresponds to a Photoionization - Auger decay - Photoionization sequence.

The demonstration of the XFEL-based two-photon photoelectron technique to study the spectroscopy of DCHs has thus been achieved. It has been applied to a series of molecules: $\mathrm{N}_{2}, \mathrm{CO}, \mathrm{N}_{2} \mathrm{O}, \mathrm{CO}_{2}$ [29] [30]. One difficulty with XFELs experiments is that they are not suited for coincidence experiments, such as the ones presented above on synchrotron sources. The reason is twofold: the low repetition rate $(120 \mathrm{~Hz}$ at LCLS compared to $\sim 1 \mathrm{MHz}$ at synchrotron) and the very high fluence which leads to the ionization of several molecules in a single laser shot. An alternative method has been developed: 'partial covariance mapping'. It is not a coincidence method but a correlation method based on a statistical analysis of ionization events. High counting rates of a few tens of events by laser shots can then be analyzed. The potentiality of this method was demonstrated with the study of DCH formation in Ne atoms [31] [32]. It reveals details of the complex electron dynamics of targets in a XFEL field, and enables one to resolve different photoionization sequences such as PAPA and PPAA. Extension of this covariance mapping method to multi dimensions was addressed theoretically by Zhaunerchyk et al [33]. They showed that three particles can be correlated but that fourfold covariance analysis fails in general. The two dimension covariance mapping was then applied to the study of aminophenol molecules [34] and of $\mathrm{C}_{2} \mathrm{H}_{2}$ and $\mathrm{C}_{2} \mathrm{H}_{6}$ [35]. 


\section{Theoretical aspects}

\subsection{Formulations of the binding energies of DCH states}

As theoretical aspects of DCH binding energies have already been described in detail [36] [37], only a brief summary will be presented here. The binding energy $B E\left(\mathrm{~K}_{i}^{-1}\right)$ of a single core-hole state whose hole is at the atomic site $i$ can be written:

$$
B E\left(\mathrm{~K}_{i}^{-1}\right)=-\varepsilon_{i}-R C\left(\mathrm{~K}_{i}^{-1}\right),
$$

where $\varepsilon_{i}$ is the Hartree-Fock energy of the core orbital and $R C\left(\mathrm{~K}_{i}^{-1}\right)$ is the generalized relaxation energy. The generalized relaxation energy includes the effects from both orbital relaxation and electron correlation, though usually the contribution from orbital relaxation is dominant. By developing the description for the single core-hole case, the binding energy of a DCH state (core holes are at the atomic sites $i$ and $j$ ) can be formulated as

$$
B E\left(\mathrm{~K}_{i}^{-1} \mathrm{~K}_{j}^{-1}\right)=-\varepsilon_{i}-\varepsilon_{j}-R C\left(\mathrm{~K}_{i}^{-1} \mathrm{~K}_{j}^{-1}\right)+R E,
$$

where $R E$ is the repulsion energy of the two core holes. The repulsion energy for a $\mathrm{K}^{-2}$ state is estimated by the analytical formula [4]

$$
R E=\left(2^{5 / 2} / 3 \pi\right)\left(Z-2^{-3 / 2}\right), \quad \text { (iii) }
$$

where $\mathrm{Z}$ is the nuclear charge of the atom on which the double core-holes is localized. As for $\mathrm{K}^{-1} \mathrm{~K}^{-1}$ states, the repulsion energy is simply approximated by the inverse of the distance $r$ between the two core-holes:

$$
R E=1 / r \text {. }
$$

The relaxation energy $R C\left(\mathrm{~K}_{i}^{-1} \mathrm{~K}_{j}^{-1}\right)$ in the DCH case, which appears in Eq. (ii), is not the simple sum of the relaxation energies on the individual core-holes, but includes a non-additive contribution $N R C\left(\mathrm{~K}_{i}^{-1} \mathrm{~K}_{j}^{-1}\right)$ upon double core-hole creation:

$$
R C\left(\mathrm{~K}_{i}^{-1} \mathrm{~K}_{j}^{-1}\right)=R C\left(\mathrm{~K}_{i}^{-1}\right)+R C\left(\mathrm{~K}_{j}^{-1}\right)+N R C\left(\mathrm{~K}_{i}^{-1} \mathrm{~K}_{j}^{-1}\right) .
$$

The non-additive contribution in the case of $\mathrm{K}^{-2}$ states with double core-holes on the same atomic site, i.e, $i=j$, is called excess relaxation energy; it reflects the local properties of the atom with the core-holes. In contrast, the non-additive contribution for $\mathrm{K}^{-1} \mathrm{~K}^{-1}$ state (i.e, $i \neq j$ ) reflects the environments of the two core-hole sites and the chemical bonds between them. It is called interatomic relaxation energy. From Eqs. (i), (ii) and (v), excess or inter-atomic relaxation energy is represented as 


$$
N R C\left(\mathrm{~K}_{i}^{-1} \mathrm{~K}_{j}^{-1}\right)=B E\left(\mathrm{~K}_{i}^{-1} \mathrm{~K}_{j}^{-1}\right)-B E\left(\mathrm{~K}_{i}^{-1}\right)-B E\left(\mathrm{~K}_{j}^{-1}\right)-R E .
$$

Since the repulsion energy can be estimated with Eq. (iii) or (iv), one can determine the excess or inter-atomic relaxation energy from the binding energies of the single and double core-hole states.

\subsection{History of the theoretical study of molecular DCH states}

The first theoretical study of molecular $\mathrm{K}^{-2}$ and $\mathrm{K}^{-1} \mathrm{~K}^{-1}$ states was presented by Cederbaum et al. in the late 1980s [4] [38] [39], a long time before the experimental observation of these states. They predicted already that the energy shifts of the DCH states are more sensitive to the chemical environment around the ionized atoms than those of the single core-hole states [4]. This pioneering study was followed by several works [40] [41] [42], although the upcoming experimental observations of DCH states were hardly foreseen at that time.

In 2009, motivated by the advent of XFEL, Santra et al. proposed in a theoretical paper, to create DCH states by sequential two-photon ionization in intense XFEL pulse [24]. Following this work, theoretical investigation of the DCH states has become extremely active in the 2010s. It has been further stimulated by the appearance of the first experimental data with XFEL and with synchrotron. Tashiro et al. calculated the binding energies of the $\mathrm{K}^{-2}$ and $\mathrm{K}^{-1} \mathrm{~K}^{-1}$ states in diatomic and triatomic molecules, as well as in the $\mathrm{C}_{2} \mathrm{H}_{2 \mathrm{n}}(\mathrm{n}=1-3)$ series, [36] [43] with the complete active space self-consistent field (CASSCF) method, and evaluated the relaxation energies for the $\mathrm{K}^{-2}$ and $\mathrm{K}^{-1} \mathrm{~K}^{-1}$ states. They showed that the interatomic relaxation energies of diatomic molecules are always negative, while in triatomic and polyatomic molecules these values are sensitive to the chemical bonds around the two core-holes. Tashiro et al. investigated also correlation satellite states associated with the DCH formation in $\mathrm{N}_{2}$ and $\mathrm{CO}$, using the restricted active space configuration interaction (RASCI) method with the state-averaged CASSCF references [17]. Symmetry breaking and hole-localization in multiple core-hole states were discussed by Carravetta and Ågren [44], based on calculation using restricted active space (RAS) SCF method.

For the calculation of DCH states in larger molecules, time-consuming methods such as CASSCF possess an obvious disadvantage due to the computational cost. To solve this problem, Takahashi et al. proposed to use the density functional theory (DFT) method for calculating DCH states. In a first step, they checked the validity of this approach by comparing the energy levels of $\mathrm{Si} \mathrm{K}$-shell DCH states of $\mathrm{SiX}_{4}(\mathrm{X}=\mathrm{H}, \mathrm{F}, \mathrm{Cl})$ obtained with the DFT and with CASSCF methods [45]. Then, they successfully applied the DFT method to nucleobases [46] and to a series of disilyl molecules bridged with hydrocarbons [47]. Interatomic relaxation energy obtained for the bridged disilyl molecules was found to change from positive to negative, when increasing the length of the central carbon bridge. Meanwhile, Kryzhevoi et al. investigated, using Green's function methods, DCH states of aminophenol 
isomers and demonstrated that the binding energies of the $\mathrm{K}^{-1} \mathrm{~K}^{-1}$ states are affected by the structural differences between the isomers [48]. In a second study, they calculated, with the $\triangle \mathrm{SCF}$ method of HF theory, the binding energies of the $\mathrm{K}^{-1} \mathrm{~K}^{-1}$ states in dicyano molecules bridged by carbon chains, and investigated the dependence of the interatomic relaxation energy on the hole-hole distance [49]. The interatomic relaxation energy is always negative and increases gradually toward zero when the distance between the two core holes increases. This general trend is in a sharp contrast to that observed in the bridged disilyl molecules [47]. Takahashi and Ueda re-investigated these two different kinds of chain molecules using both the HF and DFT methods [50]. They showed that the DFT results agree with those based on HF theory and that a fortuitous long-range correction in DFT [51] affects insignificantly the observed trends. The difference in the general trends observed for these chain molecules is understood to arise from the different environments of the two core-holes.

T. D. Thomas [52] demonstrated the usefulness of Wagner plot [53] to evaluate the initial-state effects and the relaxation energies on $\mathrm{K}^{-2}$ creations, in his study of the molecules $\mathrm{CH}_{4-\mathrm{n}} \mathrm{X}_{\mathrm{n}},(\mathrm{X}=\mathrm{F}, \mathrm{Cl} ; \mathrm{n}=1-4)$ and $\mathrm{C}\left(\mathrm{CH}_{3}\right)_{4}$. Moreover, in his paper, he introduced the generalized Wagner plots which extend this approach to analyze $\mathrm{K}^{-1} \mathrm{~K}^{-1}$ formation [52]. The generalized Wagner plot analysis has succeeded in illustrating the interatomic relaxation effects of two-site double core-hole states [54] [55] [37]. Ueda and Takahashi studied the interatomic relaxation energies in the $\mathrm{XH}_{\mathrm{m}}-\mathrm{YH}_{\mathrm{n}}(\mathrm{X}, \mathrm{Y}=\mathrm{C}, \mathrm{N}, \mathrm{O}, \mathrm{F} ; \mathrm{m}, \mathrm{n}=0-3)$ and $\mathrm{NX}_{2} \mathrm{CXO}(\mathrm{X}=\mathrm{H}, \mathrm{F})$ compounds as well as in $\mathrm{C}_{60}$, using the generalized Wagner plots of the binding energies calculated with the DFT method [54]. The $\mathrm{XH}_{\mathrm{m}}-\mathrm{YH}_{\mathrm{n}}(\mathrm{X}, \mathrm{Y}=\mathrm{C}, \mathrm{N}, \mathrm{O}, \mathrm{F} ; \mathrm{m}$, $\mathrm{n}=0-3$ ) series were soon later re-calculated with HF and CASSCF methods; they showed a clear correlation between the interatomic relaxation energy and the electron densities at the bond between the core-hole atoms [37]. Tashiro et al. systematically investigated the interatomic relaxation effects of the $\mathrm{K}^{-1} \mathrm{~K}^{-1}$ states in various molecules with polarizable unit; they used ab initio $\triangle \mathrm{SCF}$ calculations and the generalized Wagner plots approach [55].

DCH states have attracted theoretical studies to describe not only their spectroscopy but also their decay properties. The Auger decay rate of a $\mathrm{K}^{-2}$ state should be roughly twice the corresponding single core-hole rate, if we consider that the number of first-step Auger decays from a DCH state is the sum of the Auger decays from the individual core-holes. This approach has been verified in a calculation with Stieltjes imaging method [56], although it includes relaxation effects insufficiently. Inhester et al. investigated the Auger decay of the $\mathrm{K}^{-2}$ state in $\mathrm{H}_{2} \mathrm{O}$ as well as the ionization transition rate for its formation, using the single center expansion (SCE) method [23]. They found that the nuclear motion of the $\mathrm{K}^{-2}$ state affects the Auger spectrum. Subsequently they computed the Auger decay rates of the $\mathrm{K}^{-2}$ states in a sequence of isoelectronic first-row hydrides, and showed that the rates of the double core-holes are more than twice those of the single core holes [57]. This rate 
enhancement for DCH was attributed partly to an increased valence population in the vicinity of the core-holes. Tashiro et al. employed configuration interaction (CASCI) method based on the CASSCF orbitals to investigate the cascade Auger decays of the DCH states of $\mathrm{CH}_{4}, \mathrm{NH}_{3}$ and $\mathrm{H}_{2} \mathrm{CO}$ [21]. In a following study, this method for calculating the Auger spectra was applied to the decay of the $\mathrm{K}^{-2}$ states in $\mathrm{N}_{2}$ and $\mathrm{C}_{2} \mathrm{H}_{2}$ and also to the decay of the satellite states in $\mathrm{C}_{2} \mathrm{H}_{2}$, and compared with the experimental Auger spectra [22]. The detailed comparison of the experimental and theoretical Auger spectra showed a deviation only in the second-step Auger profiles, suggesting an important effect of nuclear motion of the triply charged states populated in the first-step of the Auger cascade [22]. X-ray emission spectrum from $\mathrm{K}^{-2}$ and $\mathrm{K}^{-1} \mathrm{~K}^{-1}$ states, which has not yet been observed experimentally, has also been predicted for CO [58].

\section{The related single photon simultaneous core ionization and core excitation process: $\mathbf{K}^{-2} \mathbf{V}$ states}

Closely related to double K-shell ionization, the precursor process in which one of the $\mathrm{K}$-shell electrons is ionized while the other one (from the same atom) is promoted to a vacant orbital, presents very interesting properties. For sake of conciseness, we note here those states $\mathrm{K}^{-2} \mathrm{~V}$ rather than the 2-hole 1-particle (2h-1p) notation used in ref [16] where these states were referred as pre-edge resonances below the $\mathrm{K}^{-2}$ threshold. The $\mathrm{V}$ orbital may be the LUMO or any other unoccupied orbital. There is continuous transition between $\mathrm{K}^{-2} \mathrm{~V}$ process when $\mathrm{V}$ is a Rydberg orbital and $\mathrm{K}^{-2}$ double ionization as observed a long time ago between ionization excitation process and double ionization in helium [59]. Since their first observation and identification [16], these $\mathrm{K}^{-2} \mathrm{~V}$ states have attracted a new interest due to specific properties and rich spectroscopy that will be highlighted afterwards. The $\mathrm{K}^{-2} \mathrm{~V}$ notation underlines the proximity to the $\mathrm{K}^{-2}$ ionization threshold and the pre-edge resonance assignment. However this description has no practicality for developing a theoretical model able to provide quantitative results although it presents a qualitative interest to understand some peculiarities of the $\mathrm{K}^{-2} \mathrm{~V}$ process.

Another way to describe how these states are created is to relate them to satellite excitation following $\mathrm{K}^{-1}$ inner-shell ionization. Inner-shell ionization by a high energy photon, leaves the ion in a highly excited state that will finally decay by x-ray emission (favored for high $\mathrm{Z}$ atoms) or by Auger decay (favored for low- $\mathrm{Z}$ atoms) in a typical femtosecond timescale. In the intermediate $\mathrm{K}^{-1}$ ionic state, the sudden change of the core charge strongly affects the others electrons. Their initial wave functions in the neutral are no longer eigenfunctions of the ion and a consequence is the excitation of satellite states, when this is 
energetically allowed, where K-shell ionization is accompanied by the excitation of a valence electron to a vacant orbital. This "shake-up" process results from the relaxation of valence orbitals following the change of the core charge. The excess energy relative to single core ionization is only the few $\mathrm{eV}$ necessary to excite a valence electron. The valence electron can also be promoted into the continuum -i.e. ionized- in a "shake-off" process, leading to core-valence double ionization. The transition between shake-up satellites and core-valence ionization (shake-off) can be seen in electron coincidence 2-D maps since an Auger electron is emitted in both cases due to the presence of an inner vacancy, as was first demonstrated by Hikosaka et al [60]. The relative intensity of satellite lines with respect to the main $\mathrm{K}^{-1}$ photoelectron line is typically a few percent.

Similarly, if the photon energy is high enough, it becomes also possible for the remaining 1s electron to be promoted towards a vacant orbital. This corresponds to a "super" shake-up process which is however much weaker $(\sim 1 \%)$ than valence shake-up (normal satellites) because the spatial overlap between core and valence orbital is very small and an important exchange of energy between the initially ionized 1s photoelectron and the other 1s electron is necessary to allow its excitation towards a valence orbital. Nevertheless, this process has been clearly identified thanks to very efficient electron coincidence spectroscopy with a magnetic bottle set-up. Some recently published results will be shown in the following paragraph. Moreover, since in the excitation of $\mathrm{K}^{-2} \mathrm{~V}$ states only a single photoelectron is emitted a new experimental approach to observe such states become possible that will be also discussed.

\subsection{First experimental observations of $\mathrm{K}^{-2} \mathrm{~V}$ states and their spectroscopic interest}

The first identification of $\mathrm{K}^{-2} \mathrm{~V}$ states was done by Eland et al [16] in $\mathrm{NH}_{3}$, see Fig. 12, and appeared as a by-product of $\mathrm{K}^{-2} \mathrm{DCH}$ states. The $12 \mathrm{eV}$ energy spacing, from the resonance to the $\mathrm{K}^{-2}$ edge, varying as $\mathrm{Z}^{2}$ compared to $\mathrm{K}^{-1} \mathrm{~V}$ resonances was inferred but no further analysis of the observed states was attempted. $\mathrm{K}^{-2} \mathrm{~V}$ states were then observed in $\mathrm{H}_{2} \mathrm{O}$ by Mucke et al [20].

Following our measurements of DCH process in $\mathrm{C}_{2} \mathrm{H}_{2 n}(\mathrm{n}=1,2,3)$ series, $\mathrm{N}_{2}$ and $\mathrm{CO}$ [14], we filtered out the same data set for the $\mathrm{C}_{2} \mathrm{H}_{2 n}(\mathrm{n}=1,2,3)$ series by selecting three-electron events where a single photo-electron is coincident with two Auger electrons (Hypersatellite Auger and second Auger decay) corresponding to the process :

$$
\begin{aligned}
\mathrm{hv}+\mathrm{C}_{2} \mathrm{H}_{2 \mathrm{n}} \rightarrow & \mathrm{C}_{2} \mathrm{H}_{2 \mathrm{n}}{ }^{+}\left(\mathrm{K}^{-2} \mathrm{~V}\right)+\mathrm{e}^{-} \text {Photoelectron } \\
\rightarrow & \rightarrow \mathrm{C}_{2} \mathrm{H}_{2 \mathrm{n}}{ }^{2+}\left(\mathrm{K}^{-1} \mathrm{v}^{-2} \mathrm{~V}\right)+\mathrm{e}^{-} \text {Auger Hypersatellite } \\
& \rightarrow \mathrm{C}_{2} \mathrm{H}_{2 \mathrm{n}}{ }^{3+}\left(\mathrm{v}^{-4} \mathrm{~V}\right)+\mathrm{e}^{-} \text {2nd step Auger }
\end{aligned}
$$


In Fig. 13 we show the 2D coincidence map for $\mathrm{C}_{2} \mathrm{H}_{2}$ between two electrons out of three, when the third electron is an Auger electron in the [200-260eV] energy range. On this 2D map, the $\mathrm{K}^{-2} \mathrm{~V}$ states are clearly identified by horizontal lines around $\mathrm{y}=130 \mathrm{eV}$ kinetic energy that extend on the $\mathrm{x}$ axis between 280 and $320 \mathrm{eV}$ that corresponds to the energies of hypersatellite Auger electron created in the first Auger decay of $\mathrm{K}^{-2} \mathrm{~V}$ states. Note that the energy of the hypersatellite electron extends to higher energies than in the $\mathrm{K}^{-2}$ process due to the participator Auger decay of the excited electron. The highest energy hypersatellite Auger electrons could be considered hence as a signature of the $\mathrm{K}^{-2} \mathrm{~V}$ states as hypersatellites $\mathrm{x}$-ray transitions are the signature of $\mathrm{K}^{-2}$ states [15]. By projecting the vertical band between 280 and $320 \mathrm{eV}$ of the 2D map of Fig. 13 on the vertical axis we obtain the kinetic energy (KE) of specific $\mathrm{K}^{-2} \mathrm{~V}$ photoelectrons from which the binding energies (BE) of the $\mathrm{K}^{-2} \mathrm{~V}$ states are directly deduced: $\mathrm{BE}=\mathrm{h} v-\mathrm{KE}$, and the spectra in Fig. 14. The most interesting results come from the comparison of the $\mathrm{K}^{-2} \mathrm{~V}$ spectra along the $\mathrm{C}_{2} \mathrm{H}_{2 \mathrm{n}}$ molecular series. [61]

A strong difference between the three spectra is observed with a first peak $\mathrm{A}$ around $\mathrm{BE}=$ $635 \mathrm{eV}$ which appears twice higher in $\mathrm{C}_{2} \mathrm{H}_{2}$ than in $\mathrm{C}_{2} \mathrm{H}_{4}$ and is absent for $\mathrm{C}_{2} \mathrm{H}_{6}$. The other dominant peak $\mathrm{B}$ appears comparable on the three spectra. A striking similarity appears between these spectra and the NEXAFS one for the same molecules [62] where the first peak in $\mathrm{C}_{2} \mathrm{H}_{2}$ and in $\mathrm{C}_{2} \mathrm{H}_{4}$ is due to $1 \mathrm{~s}$ excitation toward the LUMO $\pi^{*}$ orbital. This orbital is degenerated twice for $\mathrm{C}_{2} \mathrm{H}_{2}$ and does not exist in $\mathrm{C}_{2} \mathrm{H}_{6}$. This means that, at a photon energy of $770 \mathrm{eV}$, the angular momentum of the photon is not transferred to the outgoing electron but to the excited one. This situation corresponds to conjugate shake-up satellites that are also observed in $\mathrm{K}^{-1}$ ionization close to threshold [63]. The other type of satellites corresponds to direct shake-up process where the photon ionizes a K-shell electron and carries out its angular momentum (dipolar ionization), in the relaxation process the other electron is excited in a monopolar transition. $\mathrm{K}^{-1}$ satellites are mostly due to direct shake-up and conjugate shake-up is only noticeable close to threshold [63] and could be related to resonant excitation of doubly excited states [64]. The important observation is that in Fig. 14 peaks A and B have comparable intensities. Peak $\mathrm{A}$ is identified as the $\pi^{*}$ resonance resulting from conjugate shake-up while peak B is identified as a $\sigma^{*}$ resonance from direct shake-up. This is the first important result: direct and conjugate channels have similar intensities in this "super" shake-up process. This is qualitatively understandable in terms of aborted double K-shell ionization when one of the two outgoing electrons is recaptured in a vacant orbital. The angular momentum can be transferred without any preference to the ionized or to the excited electron. 
A theoretical model has been developed, that provides absolute cross sections for the $\mathrm{K}^{-2} \mathrm{~V}$ process. It was briefly presented in reference [61] and was detailed in Carniato et al [65]. It is based on extensive configuration interaction of both the initial and final state and evaluates cross sections in the dipole approximation. This model establishes the contribution of the two different processes cited above:

1- direct shake-up described by dipolar $\mathrm{K}$ shell ionization accompanied by monopolar excitation (shake-up) of the remaining $\mathrm{K}$ shell electron,

2- conjugate shake-up described by dipolar excitation of a $\mathrm{K}$ shell electron to a vacant $\mathrm{V}$ orbital accompanied by monopolar ionization (shake-off) of the remaining K-shell electron. The results of the calculations for the $\mathrm{C}_{2} \mathrm{H}_{2 n}$ series is shown in the right part of Fig. 14. It demonstrates that the direct (solid red line) and conjugate (dashed green line) contributions are indeed of similar intensity at this photon energy. It shows the unique property of $\mathrm{K}^{-2} \mathrm{~V}$ states: they can be observed with different symmetries, in contrast to the NEXAFS case where the symmetry of the $\mathrm{K}^{-1} \mathrm{~V}$ states is restricted by the dipolar excitation rules (they are thus analogous to the sole conjugate path in $\mathrm{K}^{-2} \mathrm{~V}$ states). Another interesting property of the $\mathrm{K}^{-2} \mathrm{~V}$ spectroscopy is that $\sigma^{*}$ valence orbitals which can appear as shape resonances above threshold in the $\mathrm{K}^{-1} \mathrm{~V}$ case are here found below the $\mathrm{K}^{-2}$ threshold.

\subsection{Further experimental observations of $K^{-2} V$ states}

Note that $\mathrm{K}^{-2} \mathrm{~V}$ states have not yet been observed with XFELs. One reason is that their general formation in a two-step process would need a scan of the $\mathrm{x}$-ray energy and possibly two color experiments: one can imagine for instance a $\mathrm{K} \rightarrow \mathrm{V}$ excitation in a first step with a first color (of adjustable energy) and ionization in a second step by a second photon of fixed but higher energy than the first one. Such two color experiments are not yet routinely available at XFELs. Another possibility with single color experiment would be ionization by a first photon and subsequent excitation by a second photon of the same energy. Since the second energy threshold is higher, the energy of the first photon can be sufficient to ionize and just enough to excite in the second step. Since the second step must be resonant, the process could be very efficient (such resonant process are involved in multiple ionization of rare gases and are responsible for the observation of unexpected very high charge states [66]). We can remark that equivalent $\mathrm{K}^{-1} \mathrm{~K}^{-1} \mathrm{~V}$ states exist, but their formation in a single photon process is very inefficient (similar to that of the related $\mathrm{K}^{-1} \mathrm{~K}^{-1}$ states) and they escaped detection up to now. A recent theoretical study [67] proposes that XFEls can be efficient tools to observe $\mathrm{K}^{-1} \mathrm{~K}^{-1} \mathrm{~V}$ states in heteronuclear molecules. They give the example of $\mathrm{CO}$ molecules and suggest to use two-photon processes with XFEL radiation to resonantly excite the $\mathrm{O}$ atom of the molecule in a first step and ionize the remaining $\mathrm{C}$ atom in a second step. 
Such a path should be extremely efficient because of the resonant character of the first step path.

On synchrotrons, we studied the $\mathrm{K}^{-2} \mathrm{~V}$ process in propylene molecule $\mathrm{C}_{3} \mathrm{H}_{6}$ with a single and a double bond. The $\mathrm{K}^{-2} \mathrm{~V}$ spectrum in Fig. 15a) is very similar (no background was subtracted) to what could be obtained by merely adding the spectra for $\mathrm{C}_{2} \mathrm{H}_{4}$ and $\mathrm{C}_{2} \mathrm{H}_{6}$ and the $\pi^{*}$ resonance is about one fourth in intensity of the $\sigma^{*}$ resonance.

Other experimental results have been obtained on $\mathrm{H}_{2} \mathrm{O}$ and $\mathrm{N}_{2}$ in refs. [65] [68] in which the theoretical method and formalism has been detailed. The $\mathrm{K}^{-2} \mathrm{~V}$ experimental spectrum in $\mathrm{H}_{2} \mathrm{O}$ is presented in Fig. 16 (left) and the theoretical calculation in Fig. 16 (right). Although the spectrum seems rather simple, one important result is that the first peak is broadened due to the dissociative state along the $\mathrm{H}-\mathrm{OH}$ elongation coordinate.

The $\mathrm{N}_{2}$ spectra at $979 \mathrm{eV}$ are shown in Fig. 17, with experiment in the upper-left panel and theory in the right one. It shares common features with the isoelectronic $\mathrm{C}_{2} \mathrm{H}_{2}$ molecule with the prominent and well isolated $\pi^{*}$ conjugate shake-up resonance at $877 \mathrm{eV}$ and a broader structure centered around $892 \mathrm{eV}$ which contains different resonances due to direct shake-up ( $\left.3 \mathrm{~s} \sigma, \sigma^{*} \ldots\right)$ but also to conjugate channel $(3 \mathrm{p} \pi \ldots)$. We used $\mathrm{N}_{2} \mathrm{~K}^{-2} \mathrm{~V}$ spectra to study the evolution of conjugate versus direct shake-up with photon energy to see if a faster decrease of the conjugate channel occurs. This study was limited to a photon energy range from 950 to $1150 \mathrm{eV}$ in order to keep a sufficient resolution for the magnetic bottle. The evolution of the experimental spectra give a good agreement between theory and experiment but shows only a moderate relative decrease of the conjugate channel with respect to the direct one.

Another study was performed on $\mathrm{CO}_{2}$ molecule at the carbon K-edge [69]. The advantage is that the first two resonances $\pi^{*}$ and $\sigma$ are well isolated and identified as conjugate and direct shake-up. At a photon energy of $760 \mathrm{eV}$, the ratio between the two resonances is close to 2 due to the twofold degeneracy of the first resonance. It was not possible to study this process on a broad photon range with the magnetic bottle, due to loss in absolute resolution at high energy (and to possible overlap between Carbon Auger electrons and Oxygen photoelectrons), but another type of experiment provides also some direct access to $\mathrm{K}^{-2} \mathrm{~V}$ spectra. Because only one photoelectron is emitted during the formation of $\mathrm{K}^{-2} \mathrm{~V}$ states, conventional electron spectroscopy is a possible issue to observe this process. The principle was demonstrated with the HAXPES spectrometer at the GALAXIES beamline in SOLEIL to study $\operatorname{Ar}^{+}\left(1 \mathrm{~s}^{-1} 2 \mathrm{p}^{-1} \mathrm{nl}\right)$ satellite states [70]. These are not strictly speaking $\mathrm{K}^{-2} \mathrm{~V}$ states but related $\mathrm{K}^{-1} \mathrm{~L}^{-1} \mathrm{~V}$ ones, which correspond to $\mathrm{K}$-shell ionization with simultaneous $\mathrm{L}$-shell 
excitation. Since the associated photoelectrons are well separated from "ordinary" $1 \mathrm{~s}^{-1} 31^{-1} \mathrm{nl}$ satellites (valence excitation) it was possible to extract the spectra in Fig. 18 in a few hours and to identify all the states involved. It was not possible to study $\operatorname{Ar}\left(\mathrm{K}^{-2} \mathrm{~V}\right)$ states in these condition but, in a recent experiment [71] $\mathrm{Ne}\left(\mathrm{K}^{-2} \mathrm{~V}\right)$ states have been observed and identified and reveal also a comparable contribution from direct and conjugate channels.

In the $\mathrm{CO}_{2}$ case, conventional photoelectron spectroscopy was used to observe the $\mathrm{C}\left(\mathrm{K}^{-2} \mathrm{~V}\right)$ signal at a photon energy of $2300 \mathrm{eV}$. It was necessary to accumulate during $15 \mathrm{~h}$ to observe the $\mathrm{C}\left(\mathrm{K}^{-2} \mathrm{~V}\right)$ signal on a high background due to $\mathrm{O}\left(\mathrm{K}^{-1}\right)-\mathrm{V}^{-1}$ (core-valence) double ionization. The ratio $\mathrm{C}\left(\mathrm{K}^{-2} \mathrm{~V}\right) / \mathrm{O}\left(\mathrm{K}^{-1}\right)$ is only about $10^{-5}$ but with high performance electron spectrometers and high flux synchrotron lines observing such process in nowadays possible. [69] The observed relative decrease of the conjugate channel with respect to the direct channel is found to be about a factor of 2 between 760 (i.e. $100 \mathrm{eV}$ above $\mathrm{K}^{-2}$ threshold) and $2300 \mathrm{eV}(1650 \mathrm{eV}$ above) as predicted by the theoretical model described above.

Although $\mathrm{K}^{-2} \mathrm{~V}$ process is a weak process (about $1 \%$ of $\mathrm{K}^{-1}$ ) the corresponding spectroscopy reveals interesting features when compared to normal satellites studies, XPS and NEXAFS. At fixed photon energy, both conjugate and direct "super" shake-up states appear with comparable intensities. The conjugate channel reveals resonances associated to the orbitals that are accessible in NEXAFS ( $1 \mathrm{~s} \rightarrow \mathrm{V}$ dipolar transitions). The direct shake-up gives access to orbitals with different symmetries, as in XPS. Another interesting aspect is that the resonances which appear above $\mathrm{K}^{-1}$ threshold as shape-resonances are in the $\mathrm{K}^{-2} \mathrm{~V}$ studies observed below the $\mathrm{K}^{-2}$ threshold.

\section{Conclusion}

Double photoionization from molecular inner-shell orbitals has been recently studied with multi-electron coincidence experiments and synchrotron sources. As summarized above, a large collection of results have been obtained in a few years, including the spectroscopy of these double core holes, (both the one-site $\left(\mathrm{K}^{-2}\right)$ and the two-site $\left(\mathrm{K}^{-1} \mathrm{~K}^{-1}\right)$ ones), their Auger decay and the related $\mathrm{K}^{-2} \mathrm{~V}$ process where one inner-shell electron is excited and the other is ionized. At the same time the advent of XFEL light sources has allowed complementary observation of the same processes, by creating them in a two-photon path. They confirmed the interest of the spectroscopy of DCHs which was predicted in the pioneering study by Cederbaum et al [4] in 1986, and stimulated the development of new theoretical models.

However, from a practical point of view these complementary methodologies remain technically difficult and will need further improvements to go beyond the present status. With 
synchrotron sources, the coincidence technique is able to provide high resolution spectroscopy of DCH states and this can be easily extended to $\mathrm{K}^{-2} \mathrm{~V}$ spectroscopy with up-to-date electron spectrometers and synchrotron beamlines. The real limitation comes from the very small cross-section of the order of $10^{-3}$ with respect to single K-shell ionization for $\mathrm{K}^{-2}$ states, and drops further by two orders of magnitude for $\mathrm{K}^{-1} \mathrm{~K}^{-1}$ states. The study of $\mathrm{K}^{-1} \mathrm{~K}^{-1}$ states with multi-electron coincidences on synchrotrons is thus a difficult performance. With XFEL light sources, the cross section is not an issue, and both one-site and two-site DCHs can be equally and efficiently created with short light pulses in the fs range (below the core-shell lifetime). However new experimental developments will be needed to fully disentangle all the possible processes that add up to core double ionization. With the much higher repetition rates which are planned in the new XFEL centers under construction, one should certainly consider the possibility of coincidence spectroscopy to identify clearly all the possible channels. 


\section{References}

[1] J. S. Briggs et V. Schmidt, J. Phys. B, vol. 33, p. R1, 2000.

[2] T. J. Reddish et al, Phys. Rev. Lett., p. 193001, 2008.

[3] M. S. Schöffler et al, Phys. Rev. Lett., vol. 111, p. 013003, 2013.

[4] L. S. Cederbaum, F. Tarantelli, A. Sgamellotti and J Schirmer., J. Chem. Phys., vol. 85, p. $6513,1986$.

[5] M. Piancastelli, Eur. Phys. J. Special Topics, vol. 222, p. 2035, 2013.

[6] Y. Hikosaka, P. Lablanquie, F. Penent, M. Nakano et K. Ito, Journal of Physics: Conference Series, vol. 488, p. 012012, 2014.

[7] F. Penent et al, J. Electron Spectr. Rel. Phenom., vol. 196, p. 38, 2014.

[8] N. Berrah et L. Fang, J. Electron Spectr. Rel. Phenom., vol. 204, p. 284, 2015.

[9] F. Penent et al, J. Electron Spectr. Rel. Phenom., vol. 204, p. 303, 2015.

[10] P. Kruit et F. H. Read, J. Phys. E, vol. 16, p. 313, 1983.

[11] J. H. D. Eland, O. Vieuxmaire, T. Kinugawa, P. Lablanquie, R. I. Hall and F. Penent, Phys. Rev. Lett., vol. 90, p. 053003, 2003.

[12] P. Lablanquie et al, Phys.Rev.Lett., vol. 106, p. 069003, 2011.

[13] R. Wehlitz et al, Phys. Rev. Lett., vol. 67, p. 3764, 1991.

[14] M. Nakano et al, Phys.Rev.Lett., vol. 110, p. 163001, 2013.

[15] J. Hoszowska et J.-C. Dousse, J. Elect. Spectr. Related Phenomena, vol. 188, p. 62, 2013.

[16] J. Eland et al, Phys.Rev.Lett., vol. 105, p. 213005, 2010.

[17] M. Tashiro, K. Ueda et M. Ehara, «DCH Satellite spectra N2 CO,»Chem. Phys. Lett., vol. 521, p. 45, 2012.

[18] P. Lablanquie et al, Phys.Rev.Lett., vol. 107, p. 193004, 2011.

[19] L. Hedin et al, J.Chem.Phys., vol. 140, p. 044309, 2014.

[20] M. Mucke et al, Chem.Phys.Lett., vol. 558, p. 82, 2013.

[21] M. Tashiro, K. Ueda et M. Ehara, J. Chem. Phys., vol. 135, p. 154307, 2011.

[22] M. Tashiro et al, J. Chem. Phys. , vol. 137, p. 224306, 2012.

[23] L. Inhester, C. F. Burmeister, G. Groenhof et H. Grubmüller, J. Chem. Phys. , vol. 136, p. 144304, 2012.

[24] S. Santra, N. V. Kryzhevoi et L. S. Cederbaum, Phys.Rev.Lett., vol. 103, p. 013002, 2009. 
[25] L. Young et al, Nature, vol. 466, p. 56, 2010.

[26] J. P. Cryan et al, Phys.Rev.Lett., vol. 105, p. 083004, 2010.

[27] L. Fang et al, Phys.Rev.Lett, vol. 105, p. 083005, 2010.

[28] N. Berrah et al, Proc.Nat.Acad.Sci.USA, vol. 108, p. 16912, 2011.

[29] P. Salèn et al, Phys.Rev.Lett., vol. 108, p. 153003, 2012.

[30] M. Larsson et al, J.Phys.B-At.Mol.Opt.Phys, vol. 46, p. 164030, 2013.

[31] L. J. Fransinski et al, Phys.Rev.Lett., vol. 111, p. 073002, 2013.

[32] V. Zhaunerchyk et al, J. Phys. B: At. Mol. Opt. Phys., vol. 46, p. 164034, 2013.

[33] V. Zhaunerchyk, L. J. Frasinski, J. Eland and R. Feifel, Phys.Rev.A, vol. 89, p. 053418, 2014.

[34] V. Zhaunerchyk et al, J. Phys. B, vol. 48, p. 244003, 2015.

[35] M. Mucke et al, New J. Phys., vol. 17, p. 073002, 2015.

[36] M. Tashiro et al, J.Chem.Phys., vol. 132, p. 184302, 2010.

[37] O. Takahashi, N. V. Kyrzhevoi et K. Ueda, J. Elec. Spect. Rel. Phen., vol. 204, p. 290, 2015.

[38] L. S. Cederbaum, Phys. Rev. A, vol. 35, p. 622, 1987.

[39] L. S. Cederbaum, F. Tarantelli, A. Sgamellotti and J. Schirmer, J. Chem. Phys., vol. 86, p. 2168, 1987.

[40] E. M. Ohrendorf, L. S. Cederbaum et F. Tarantelli, Phys. Rev. A, vol. 44, p. 205, 1991.

[41] H. Agren et H. J. Jensen, Chem. Phys., vol. 172, p. 45, 1993.

[42] C. Reynaud et al, J. Phys. B, vol. 29, p. 5403, 1996.

[43] M. Tashiro, M. Ehara et K. Ueda, Chem. Phys. Lett., vol. 496, p. 217, 2010.

[44] V. Carravetta et H. Agren, J. Phys. Chem. A, vol. 117, p. 6798, 2013.

[45] O. Takahashi, M. Tashiro, M. Ehara, K. Yamasaki et K. Ueda, Chem. Phys., vol. 384, p. $28,2011$.

[46] O. Takahashi, M. Tashiro, M. Ehara, K. Yamasaki et K. Ueda, J. Phys. Chem. A, vol. 115, p. 12070, 2011.

[47] O. Takahashi, K. Yamasaki, S. Nagaoka et K. Ueda, Chem. Phys. Lett., vol. 518, p. 44, 2011.

[48] N. V. Kryzhevoi, R. Santra et L. S. Cederbaum, J. Chem. Phys. , vol. 135, p. 084302, 2011.

[49] N. V. Kryzhevoi, M. Tashiro, M. Ehara et L. S. Cederbaum, J. Chem. Phys., vol. 137, p. 154316, 2012. 
[50] O. Takahashi et K. Ueda, Chem. Phys., vol. 440, p. 64, 2014.

[51] H. Iikura, T. Tsuneda, T. Yanai et K. Hirao, J. Chem. Phys., vol. 115, p. 3540, 2001.

[52] T. D. Thomas, J. Phys. Chem. A, vol. 116, p. 3856, 2012.

[53] G. Moretti, J. Electron Spectr. Rel. Phenom., vol. 95, p. 95, 1998.

[54] K. Ueda et O. Takahashi, J. Electron Spectr. Rel. Phenom., vol. 185, p. 301, 2012.

[55] M. Tashiro, N. V. Kryzhevoi, L. S. Cederbaum et M. Ehara, J. Phys. B: At., Mol. Opt. Phys. , vol. 46, p. 164012, 2013.

[56] P. Kolorenc et V. Averbukh, J. Chem. Phys., vol. 135, p. 134314, 2011.

[57] L. Inhester, G. Groenhof et H. Grubmüller, J. Chem. Phys., vol. 138, p. 164304, 2013.

[58] M. Tashiro, J. Electron Spectr. Rel. Phenom., vol. 189S, p. 19, 2013.

[59] R. Wehlitz et al, J. Phys. B, vol. 30, p. L51, 1997.

[60] H. Hikosaka, T. Aoto, P. Lablanquie, F. Penent, E. Shigemasa et K. Ito, Phys. Rev. Lett., vol. 97, p. 053003, 2006.

[61] M. Nakano et al, Phys.Rev.Lett., vol. 111, p. 123001, 2013.

[62] J. Stöhr, NEXAFS Spectroscopy, Berlin: Springer Series in Surface Science Vol. 25 (Springer-Verlag), 1992.

[63] B. Kempgens et al, J. Chem. Phys., vol. 107, p. 4219, 1997.

[64] L. Ungier et T. D. Thomas, Phys. Rev. Lett., vol. 53, p. 435, 1984.

[65] S. Carniato et al, J.Chem.Phys., vol. 142, p. 014307, 2015.

[66] B. Rudek et al, Nature Photonics, vol. 6, p. 858, 2012.

[67] P. V. Demekhin et L. S. Cederbaum, J. Phys. B, vol. 46, p. 164008, 2013.

[68] S. Carniato et al, J.Chem.Phys., vol. 142, p. 014308, 2015.

[69] S. Carniato et al, submitted.

[70] R. Püttner et al, Phys. Rev. Lett., vol. 114, p. 093001, 2015.

[71] G. Goldsztejn et al, submitted. 

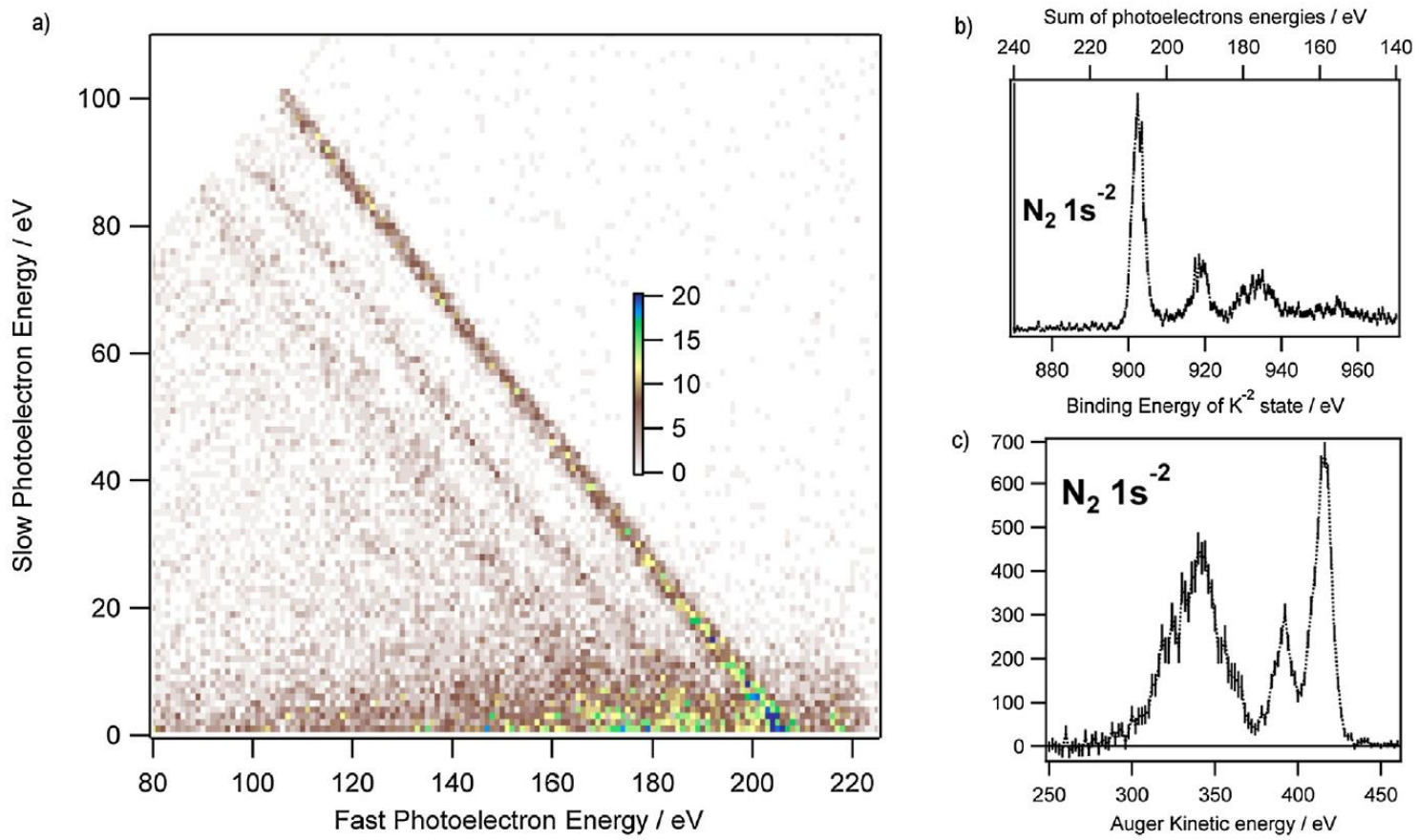

Fig.1: Single photon 1-site core double photoionization of $\mathrm{N}_{2}$ [12], obtained at a photon energy of $1110 \mathrm{eV}$. The spectra have been extracted from quadruple coincidence events in which one detects the two photoelectrons and the two Auger electrons emitted in the double core ionization and decay. (a) Energy correlation between the two photoelectrons, when detected in coincidence with two Auger electrons chosen respectively in the ranges [300-375eV] and [375-450eV]. The diagonal lines correspond to the different DCH states. (b) Spectrum of the $\mathrm{K}^{-2}$ states, obtained from the histogram of the sum of the energies of the two photoelectrons in (a). (c) Energy distribution of the Auger electrons emitted upon decay of the $\mathrm{K}^{-2}$ ground state. Reprinted with permission from [9] 


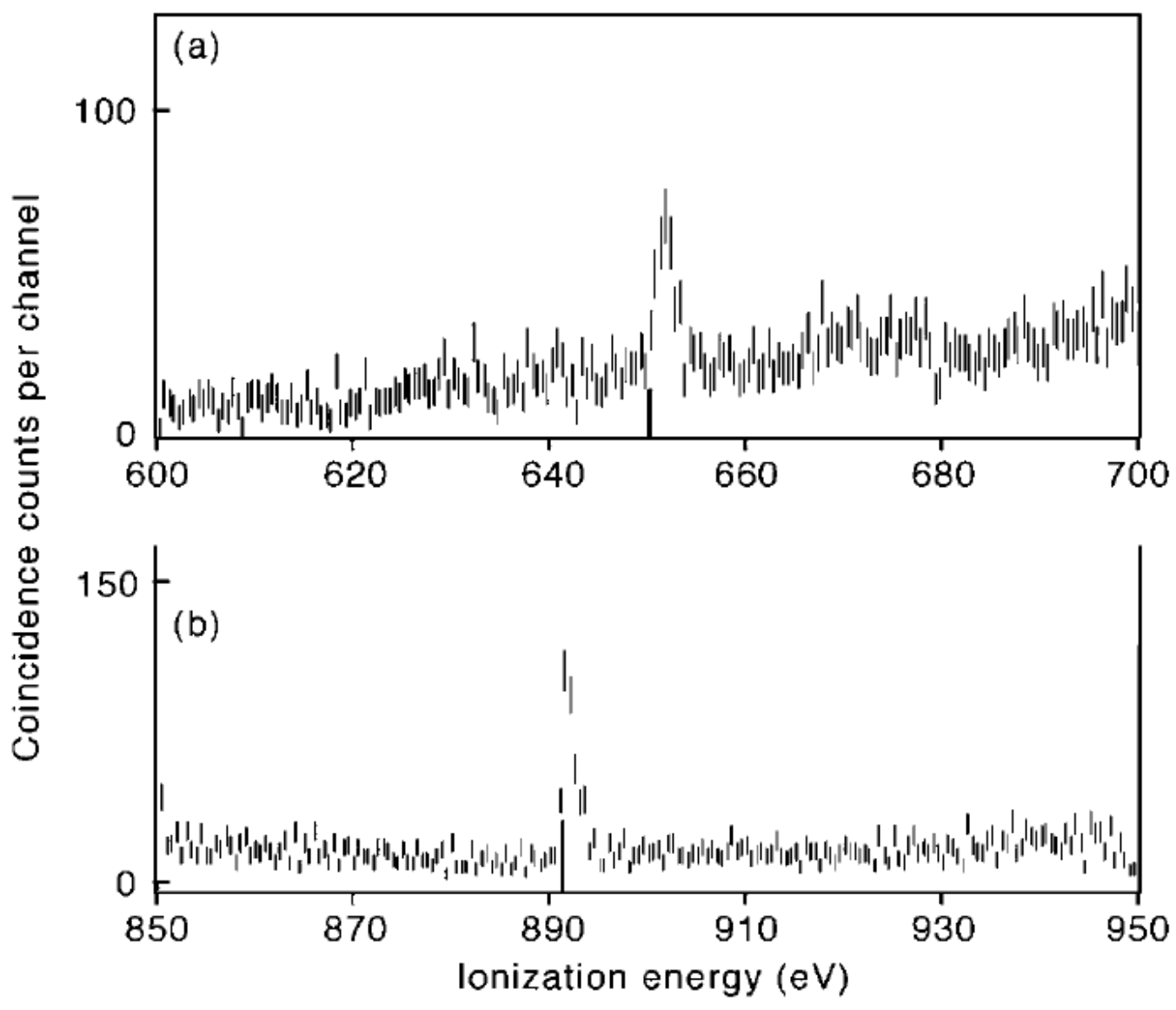

Fig. 2 Spectrum of the $\mathrm{K}^{-2}$ Double core hole states in $\mathrm{CH}_{4}$ (a) and $\mathrm{NH}_{3}$ (b) obtained respectively at a photon energy of 750 and $950 \mathrm{eV}$. The spectra have been extracted from triple coincidence data. Theoretical DCH levels are shown as bars for direct comparison. Reprinted with permission from [16] 

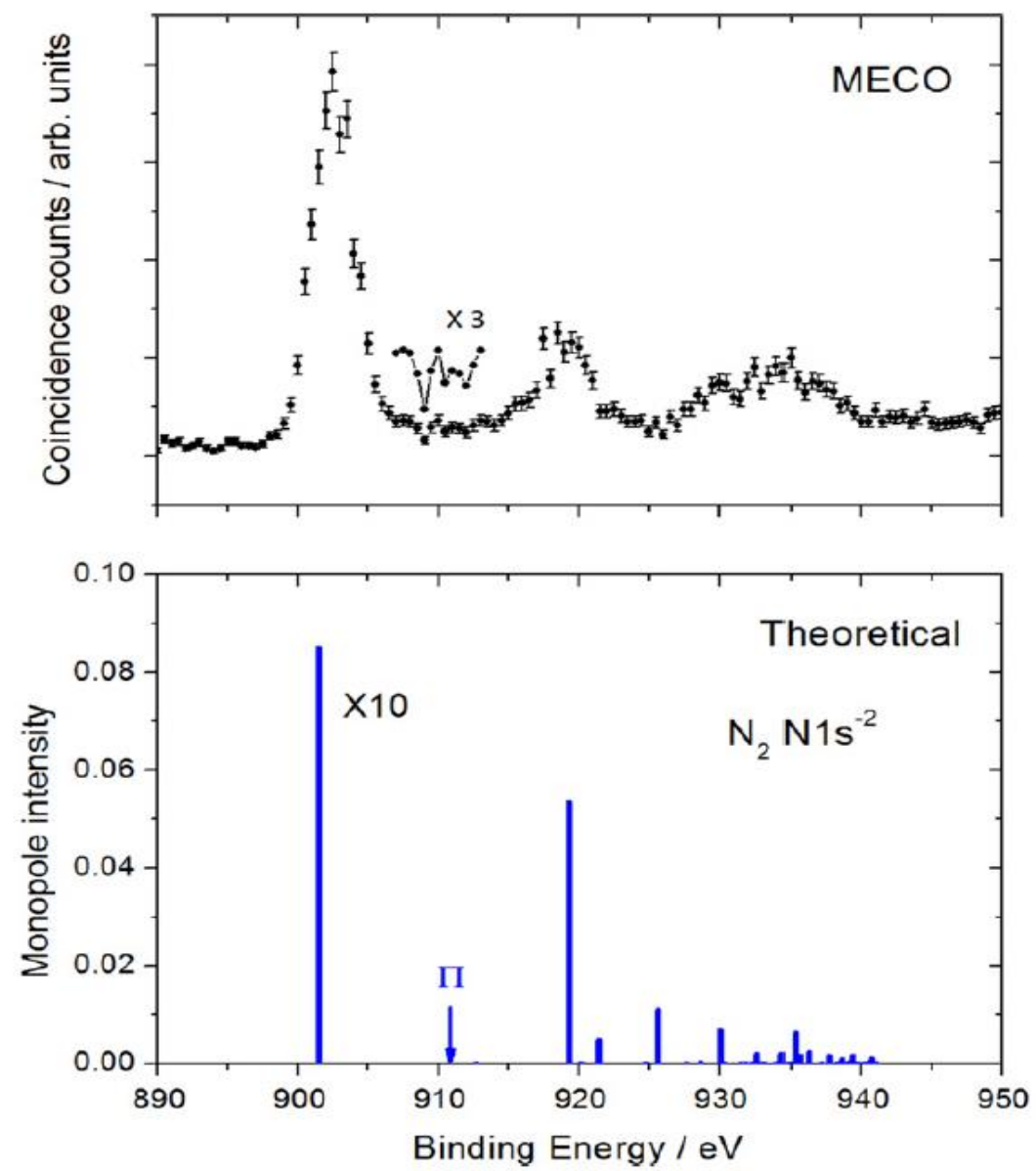

Fig. 3 : Spectrum of the $\mathrm{K}^{-2}$ Double core hole states in $\mathrm{N}_{2}$ (upper part) showing the main line and satellite states and their calculations by Tashiro et al [17]. MECO stands for 'Multi Electron Coincidences' experiments which are the ones from Fig. 1b) Reprinted with permission from [17] 


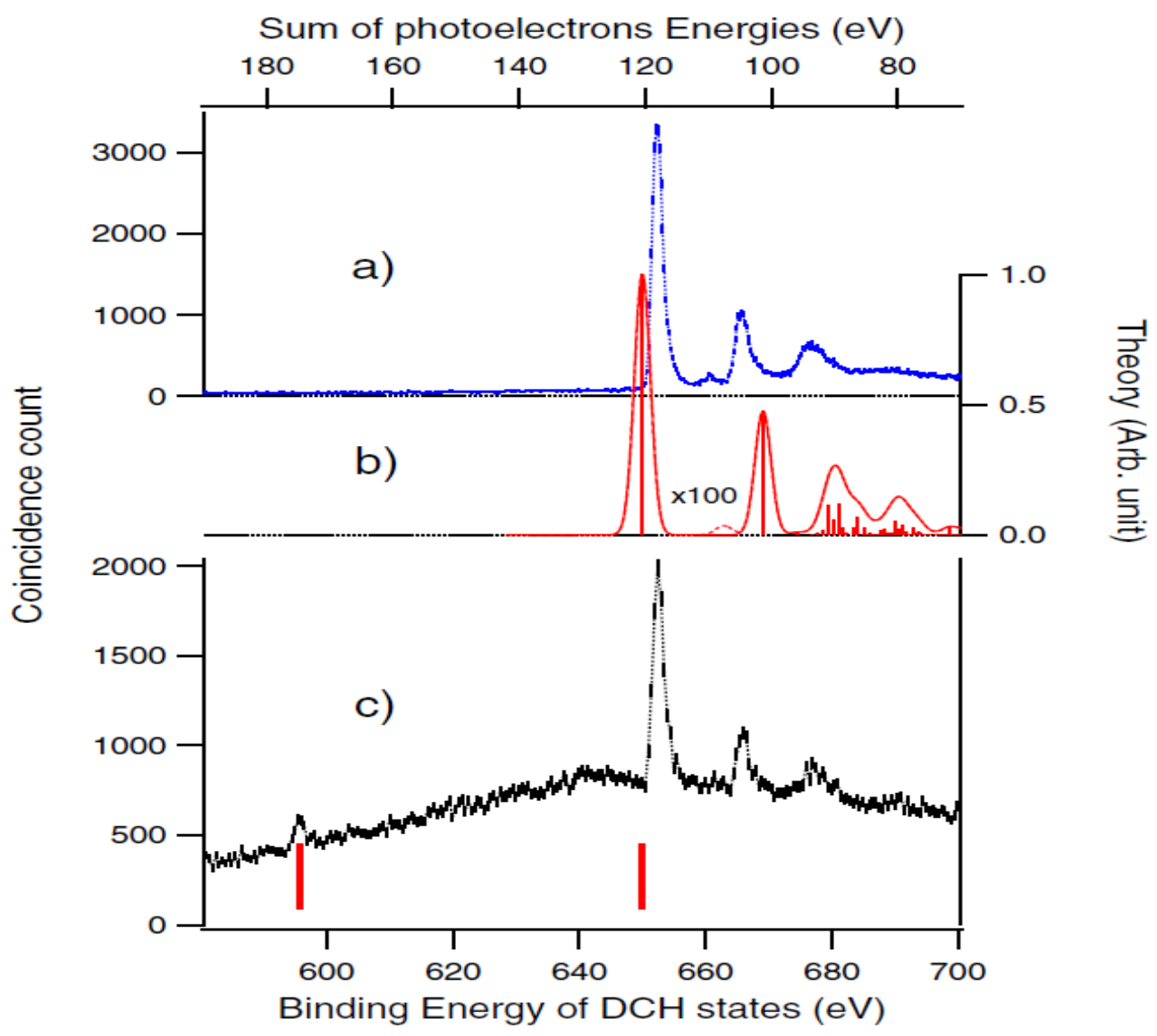

Fig. 4 : 1-site and 2-site DCHs in $\mathrm{C}_{2} \mathrm{H}_{2}$. (a) Spectrum of the $\mathrm{K}^{-2}$ Double core hole states in $\mathrm{C}_{2} \mathrm{H}_{2}$, obtained from quadrupole coincidence data, and from the sum of the energies of the two photoelectrons when detected in coincidence with the two released Auger electrons of 200-270 and 270-320 eV. (b) Predictions for $\mathrm{K}^{-2} \mathrm{DCH}$ spectrum. The $\times 100$ magnifying factor applies to the portion of the theoretical spectrum between the two main peaks to visualize the position of the lowest energy satellite state (dotted line). (c) is similar to (a) but here triple coincidence events are selected, and one plots the sum of the energies of the two photoelectrons when detected in coincidence with only one Auger electron of $230-250 \mathrm{eV}$. The one site $\mathrm{K}^{-2}$ Double core hole states continue to be selected, but the two site $\mathrm{K}^{-1} \mathrm{~K}^{-1}$ Double core hole state signal appears at a binding energy of $595.6 \mathrm{eV}$. Electrons of less than $10 \mathrm{eV}$ in (a) and (c) and of more than $60 \mathrm{eV}$ in (c) have been discarded to reduce background. Vertical bars at the bottom correspond to theoretical calculations for the position of the $\mathrm{K}^{-1} \mathrm{~K}^{-1}$ and $\mathrm{K}^{-2}$ main lines. A photon energy of $\mathrm{h} v=770.5 \mathrm{eV}$ was used. Reprinted with permission from [18] 


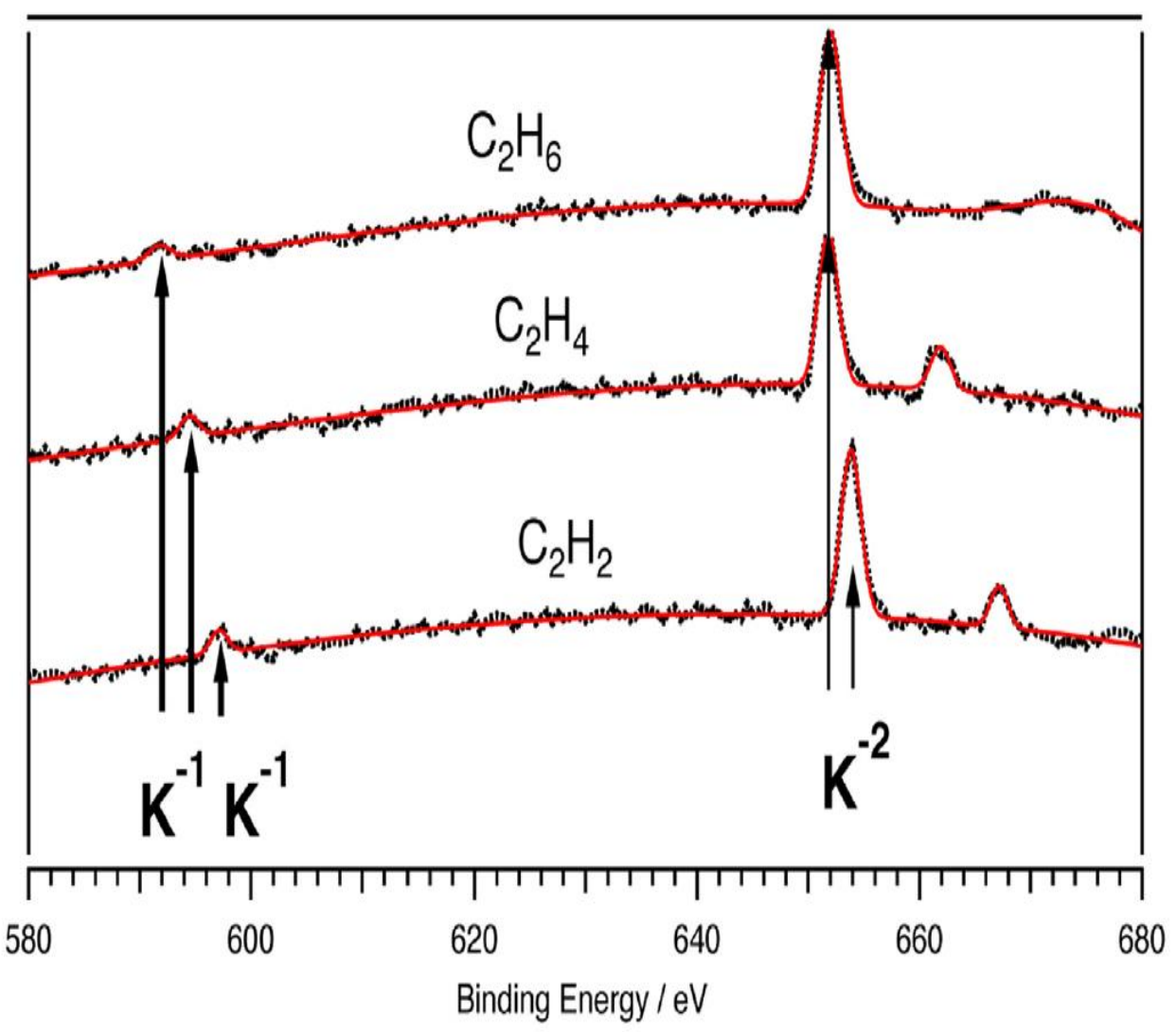

Fig. 5: Observation of $\mathrm{K}^{-1} \mathrm{~K}^{-1}$ Double core holes states in the $\mathrm{C}_{2} \mathrm{H}_{2 \mathrm{n}}$ series. As in Fig. 4(c), spectra are obtained from triple coincidence data, and from the sum of the energies of the two photoelectrons when detected in coincidence with only one Auger electron of 230-250 eV. A photon energy of hv $=$ $770 \mathrm{eV}$ was used. The red lines show the fit of our experimental data for the $\mathrm{K}^{-1} \mathrm{~K}^{-1}$ and $\mathrm{K}^{-2}$ peaks by Gaussian functions and a cubic background. Reprinted with permission from [9]. 


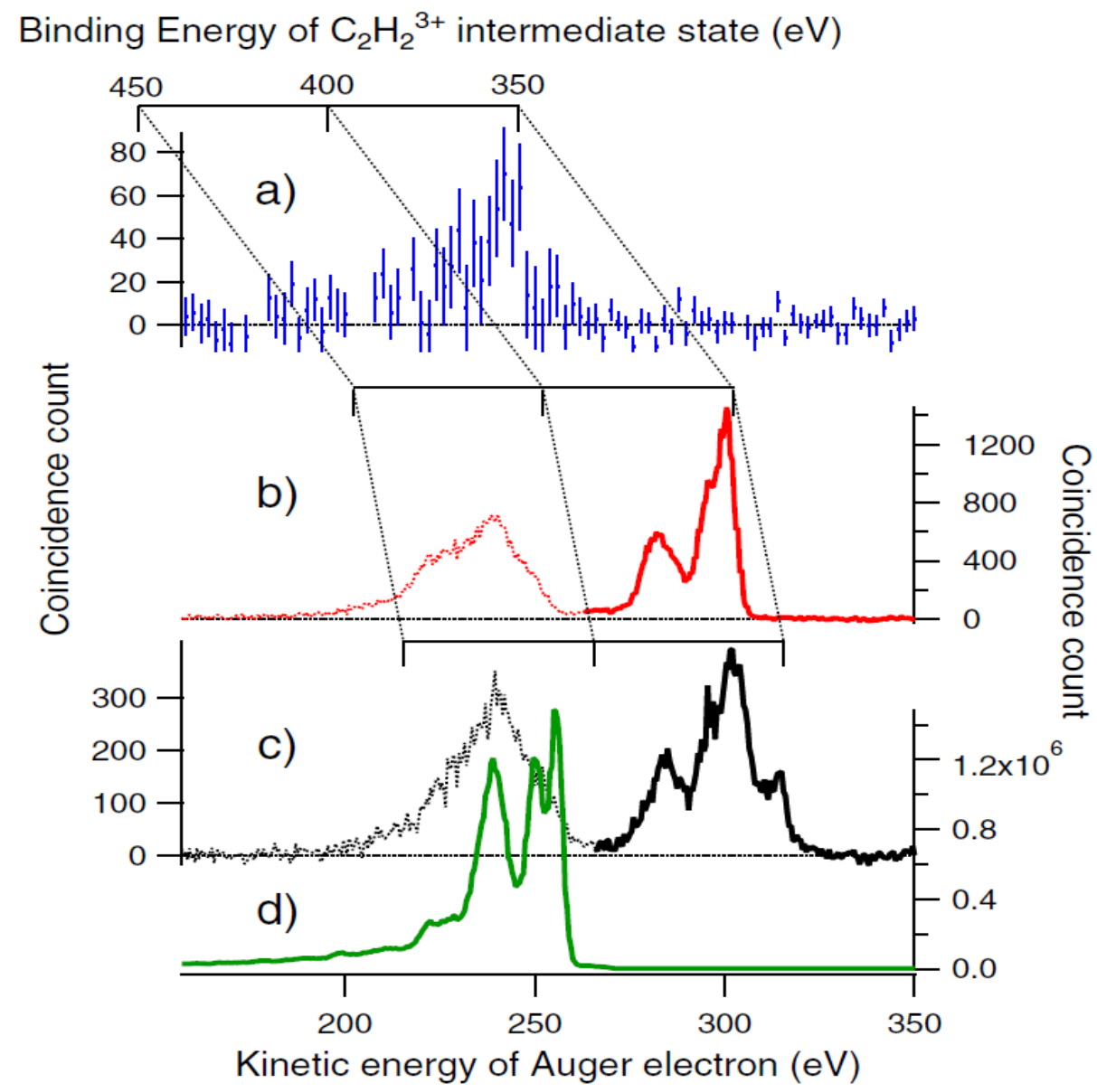

Fig. 6 Auger spectra associated with the Auger decays of: the main $\mathrm{K}^{-1} \mathrm{~K}^{-1}$ (a) and $\mathrm{K}^{-2}$ (b) states in $\mathrm{C}_{2} \mathrm{H}_{2}$. They are compared with the Auger spectra of the most intense $\mathrm{K}^{-2}$ satellite state (c) and of the single $\mathrm{K}$-shell core hole (d). In (b) and (c) the solid line displays the first emitted hypersatellite Auger electron, while the dotted line corresponds to the second emitted, satellite Auger electron; the top scale applies only for the first hypersatellite Auger electron (solid line) and reveals the binding energy of the $\mathrm{C}_{2} \mathrm{H}_{2}{ }^{3+}$ intermediate states populated in the first step of the DCH Auger decay (Equation $1 b$ ) and $2 b$ ). Reprinted with permission from [18]. 


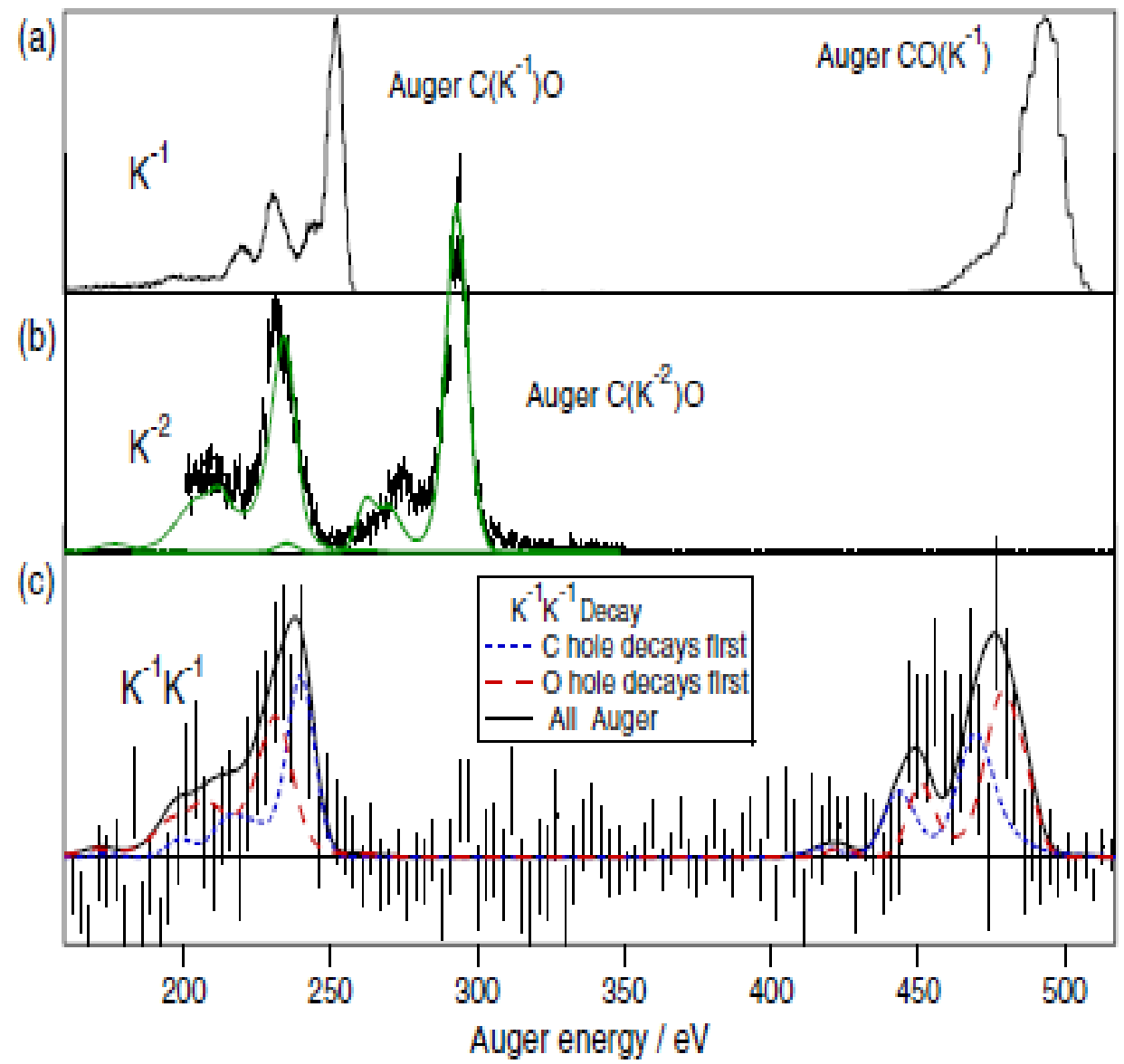

Fig. 7: Auger spectra associated with the decays of: (b) $\mathrm{C} \mathrm{K}^{-2}$ and (c) $\mathrm{K}^{-1} \mathrm{~K}^{-1}$ states in CO. They are compared with the Auger spectra of the single K-shell $\mathrm{C}$ and $\mathrm{O}$ core hole (a). The solid lines in (b) and (c) represent the calculated Auger spectra. For the $\mathrm{K}^{-1} \mathrm{~K}^{-1}$ decay (c), two contributions of similar weight are predicted, in which it is either the $\mathrm{C} 1 \mathrm{~s}$ hole (blue dots) or the $\mathrm{O} 1 \mathrm{~s}$ hole (red hyphens) that decays first. Reprinted with permission from [14] 

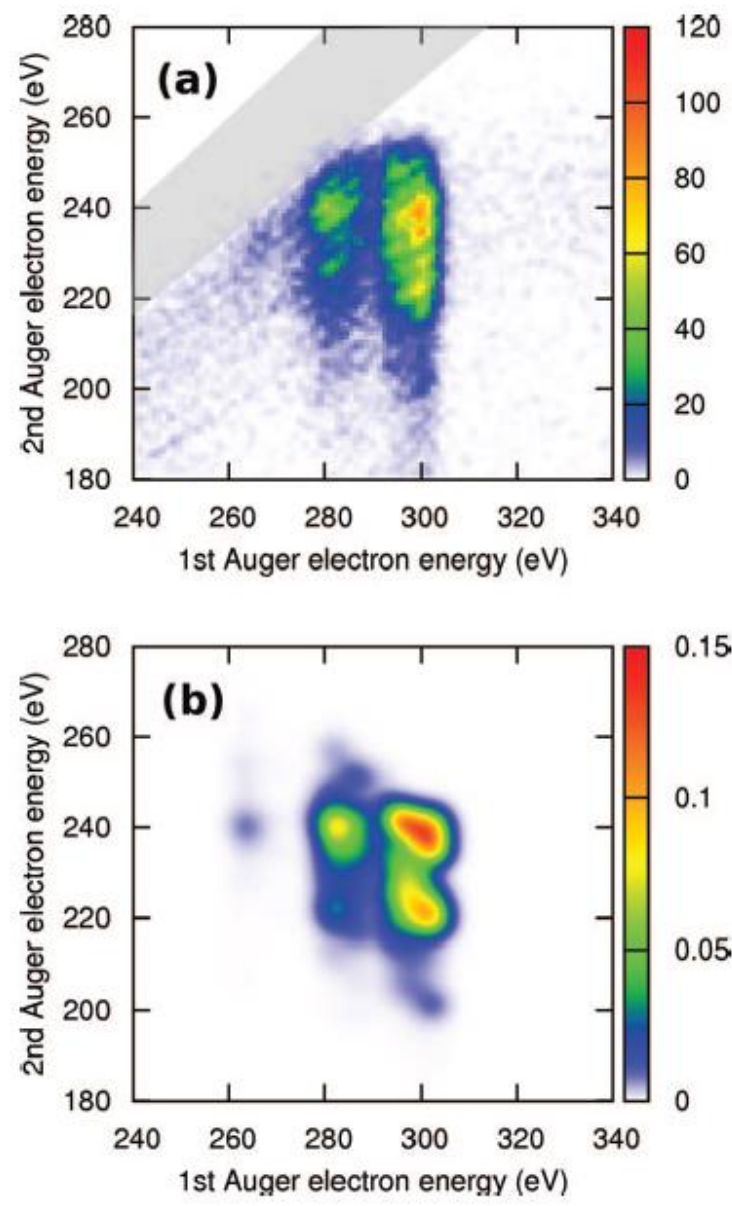

Fig. 8: Auger decay of $\mathrm{K}^{-2}$ DCHs in $\mathrm{C}_{2} \mathrm{H}_{2}$ : two-dimensional energy correlation map of the two Auger electrons emitted upon the decay of the $\mathrm{C}_{2} \mathrm{H}_{2} \mathrm{~K}^{-2}$ ground state. (a) is experiment, (b) theory. In (a) the faster Auger electron is reported on the $\mathrm{x}$ axis, while the slower one is on the y axis. Experiment is blind in the shaded zone, which corresponds to electron pairs of too close energies, so that their successive arrival at the detector falls within its $15 \mathrm{~ns}$ dead time. In (b) the $\mathrm{x}$ axis corresponds to the first electron emitted in the Auger decay, while the y axis corresponds to the second Auger electron. Reprinted with permission from [22] 

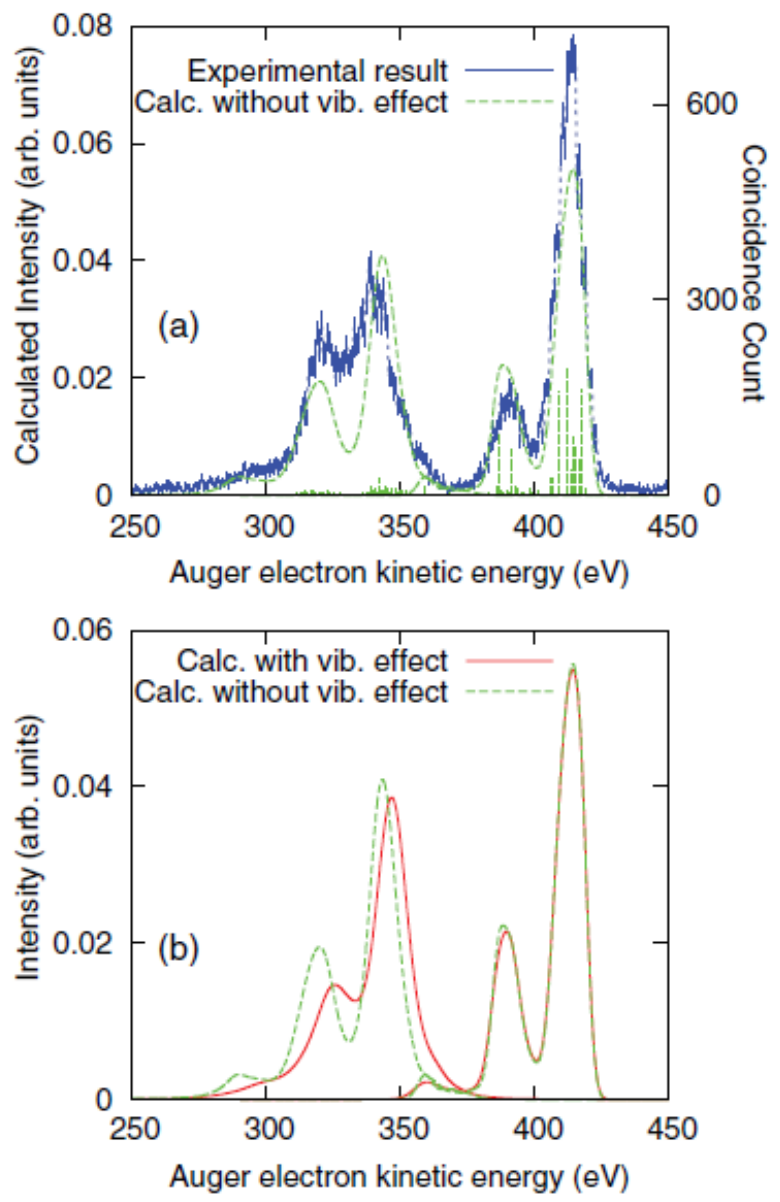

Fig. 9: Auger spectra associated with the decays of $\mathrm{K}^{-2} \mathrm{DCH}$ ground state in $\mathrm{N}_{2}$. (a) compares experiment with calculation at fixed nuclear distances. (b) shows the effect of nuclear motion on the calculated Auger spectrum. Reprinted with permission from [22] 


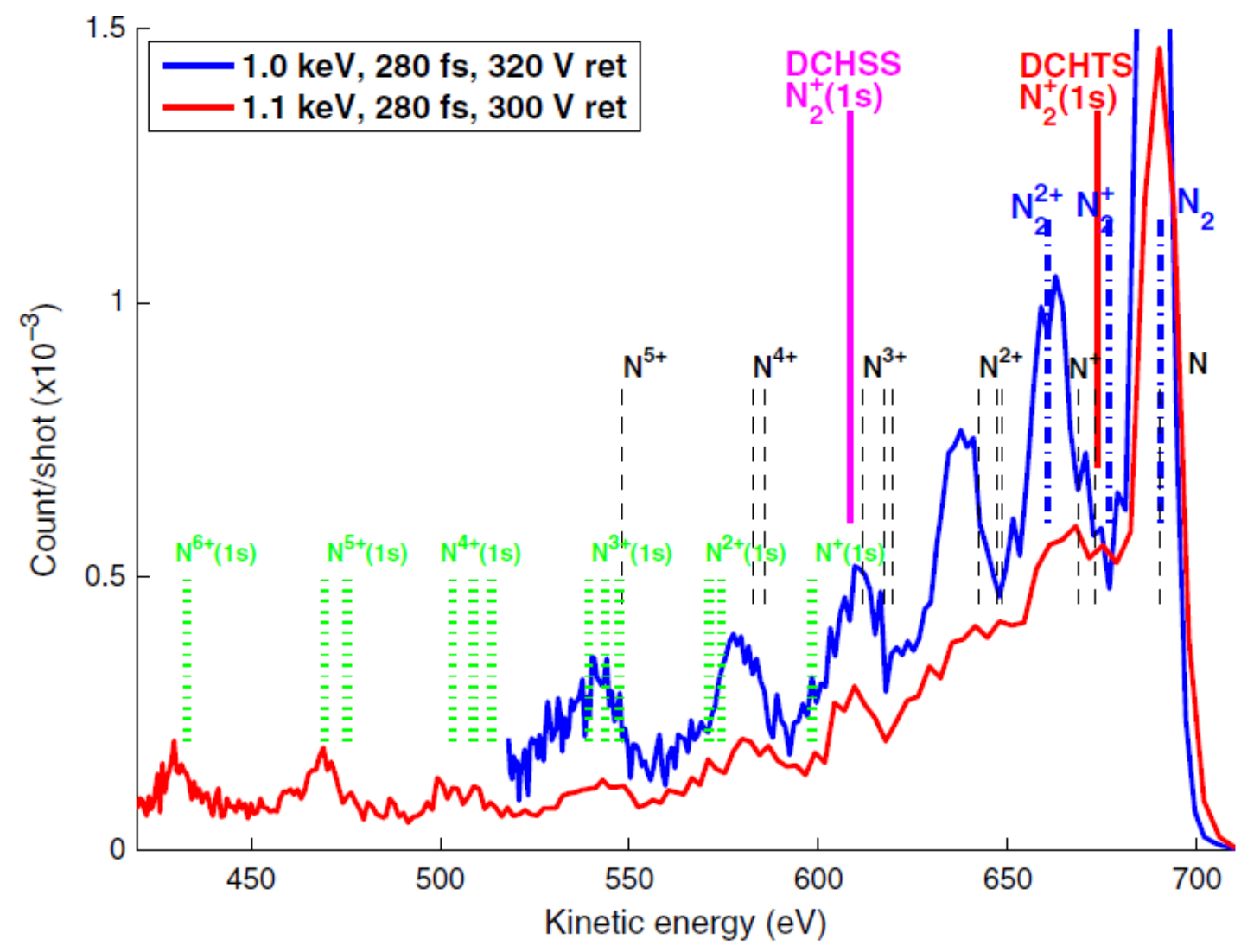

Fig.10: Photoelectron spectra recorded with XFEL radiation of $1.1 \mathrm{keV}$ and $1.0 \mathrm{keV}$. The electron energies in the $1 \mathrm{keV}$ spectrum have been shifted by $100 \mathrm{eV}$ to higher kinetic energies. Vertical markers indicate the calculated photoelectron line positions for various molecular and atomic states of $\mathrm{N}_{2}$. Reprinted with permission from [27] 


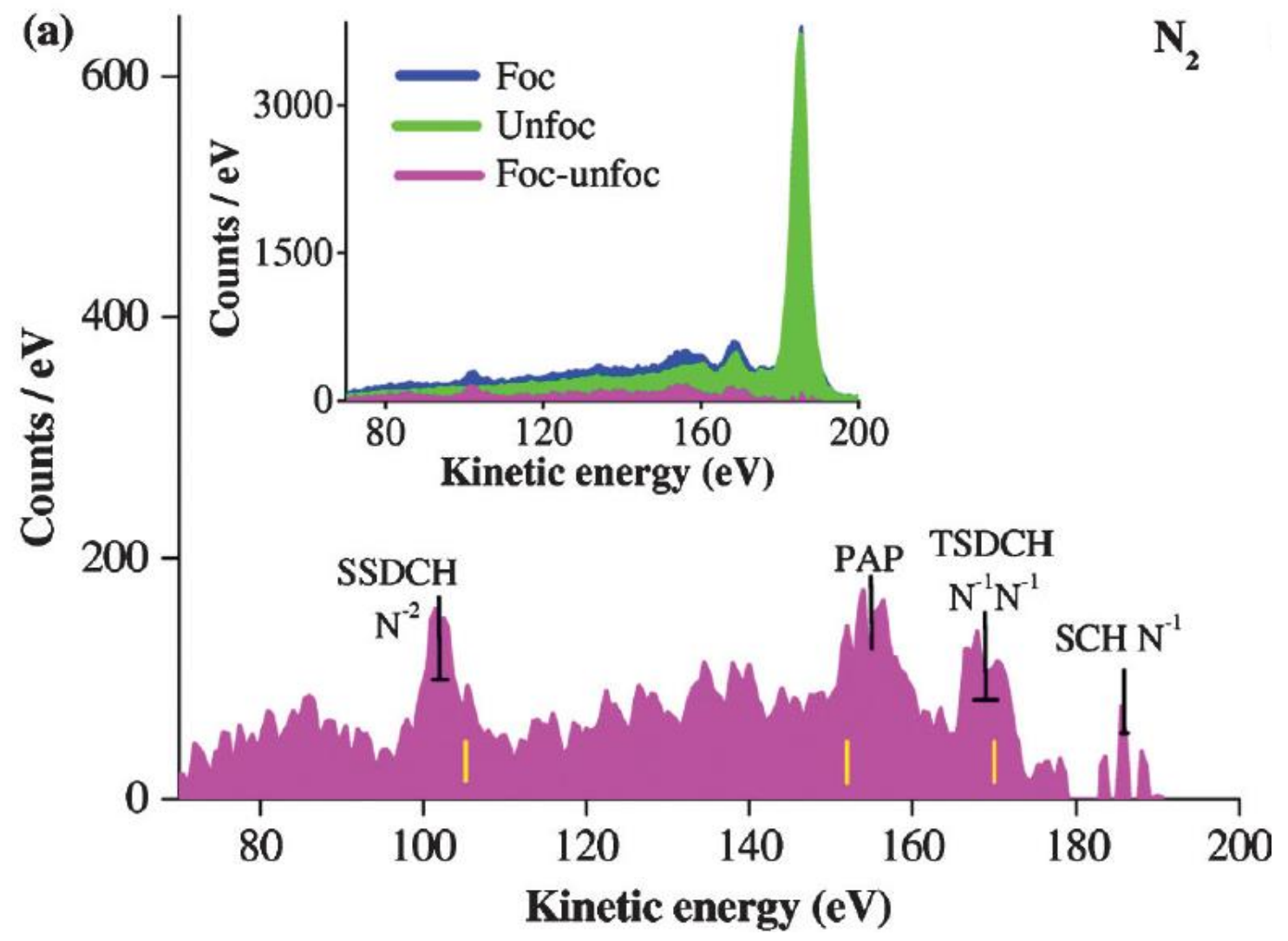

Fig 11: Photoelectron spectra of $\mathrm{N}_{2}$ recorded with XFEL radiation of 596eV. Blue curve: focused x-rays; green curve unfocused; spectra are normalized at the $\mathrm{SCH}$ peak. Magenta curve: difference spectra. Black vertical lines: experimental energies of the $\mathrm{SCH}, \mathrm{DCH}$, and PAP features; horizontal lines: estimated errors; yellow vertical lines: theoretical energies. Reprinted with permission from [29] 


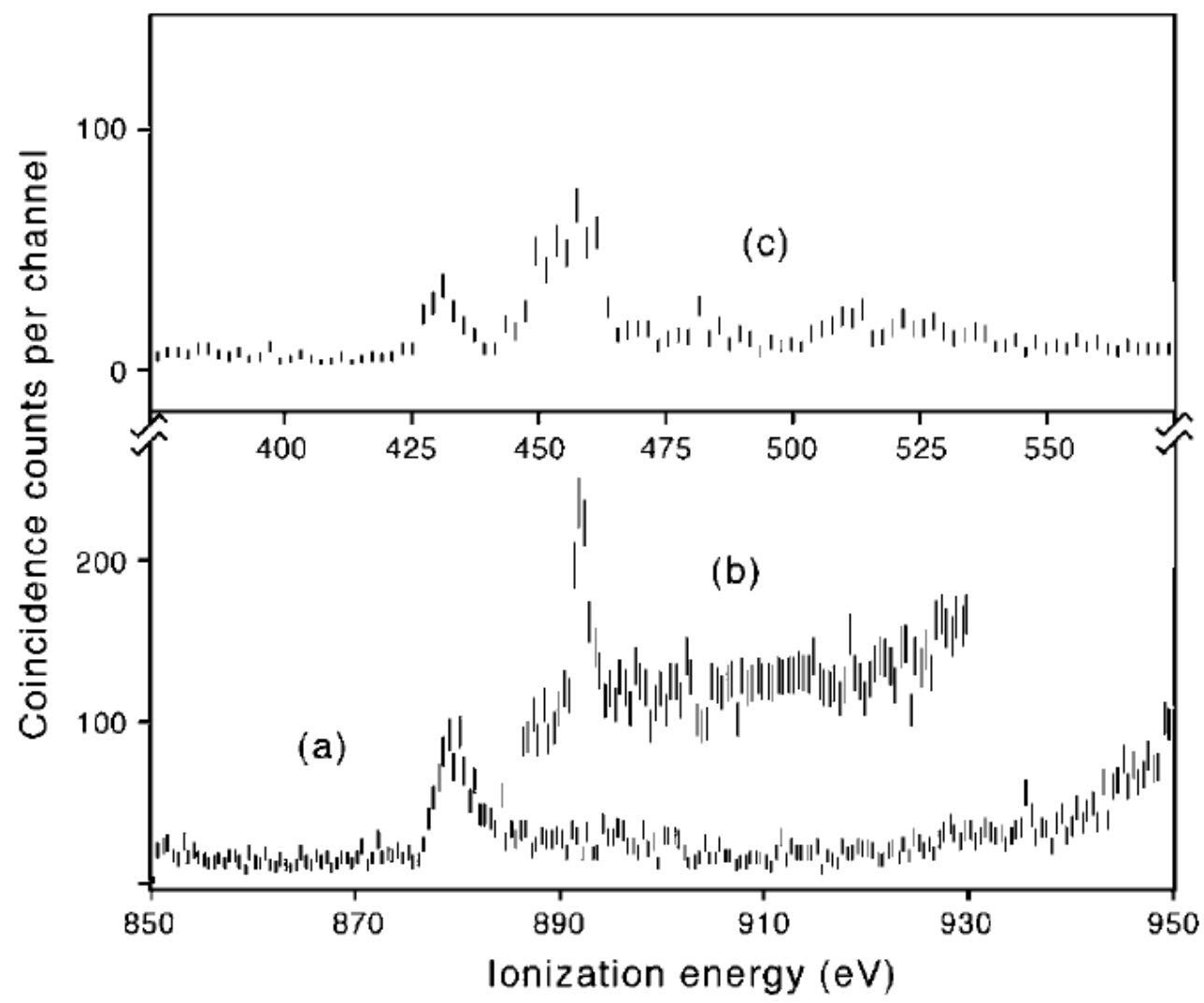

Fig 12: Evidence of the formation of $\mathrm{K}^{-2} \mathrm{~V}$ states in $\mathrm{NH}_{3}$. (a) shows the $\mathrm{K}^{-2} \mathrm{~V}$ singly ionized states from twofold coincidences, compared with a simply filtered DCH spectrum (b) -see Fig 2(b)-. (c) Spectrum of doubly charged products in coincidence with the $\mathrm{K}^{-2} \mathrm{~V}$ peak at $880 \mathrm{eV}$ shown in (a). The peak at 432 $\mathrm{eV}$ represents direct core-valence ionization which occurs for all energies of the electrons, while the peak at $456 \mathrm{eV}$ appears only in coincidence with the $\mathrm{K}^{-2} \mathrm{~V}$ photoelectron peak. Reprinted with permission from [16] 


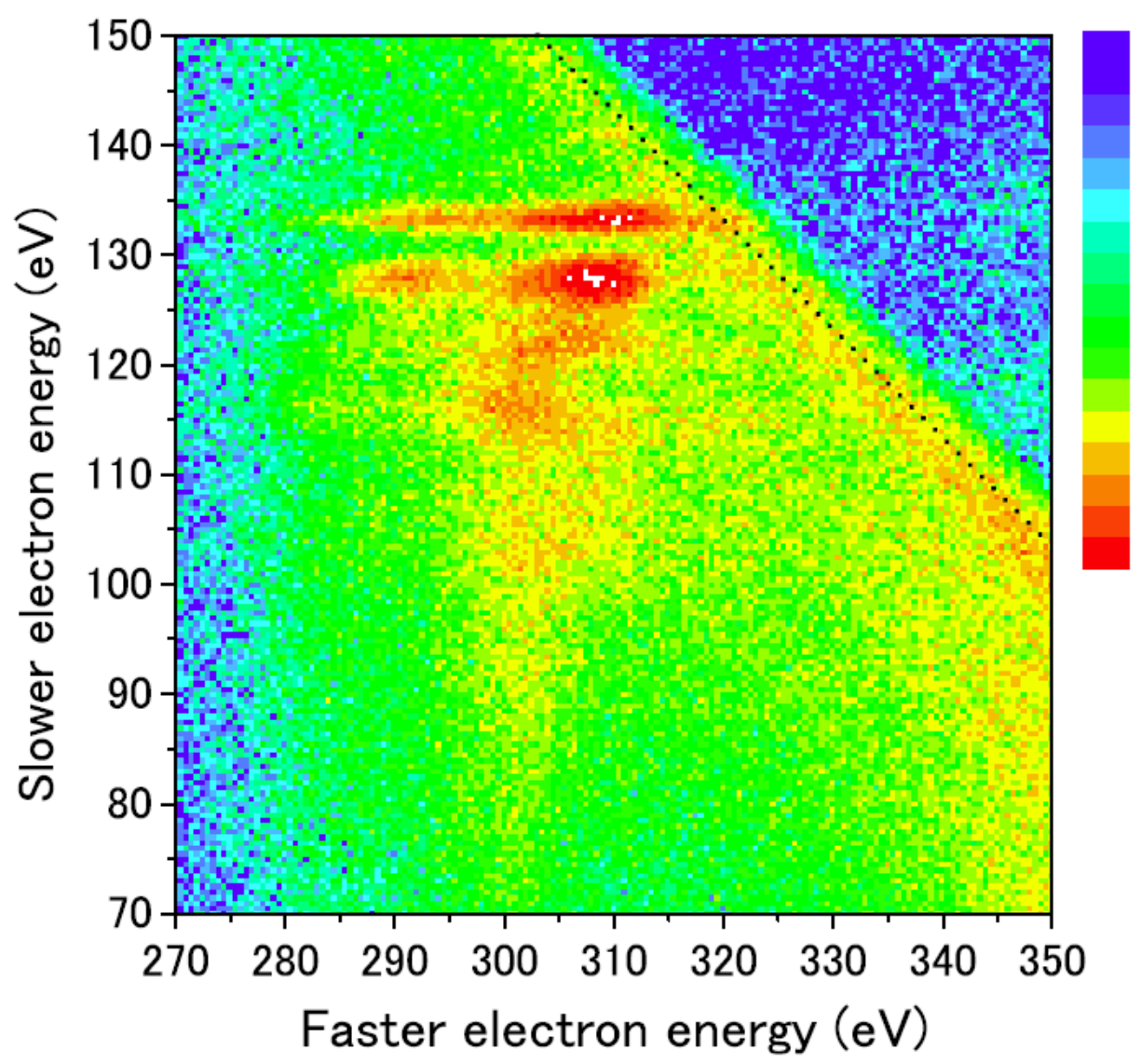

Fig. 13: Evidence of the formation of $\mathrm{K}^{-2} \mathrm{~V}$ states in $\mathrm{C}_{2} \mathrm{H}_{2}$. Energy correlation between two electrons detected in coincidence with a third one of (200-260) eV at a photon energy of 770 $\mathrm{eV}, \sim 118 \mathrm{eV}$ above the double core hole threshold [14]. Only three electron events are considered here. Reprinted with permission from [61] 

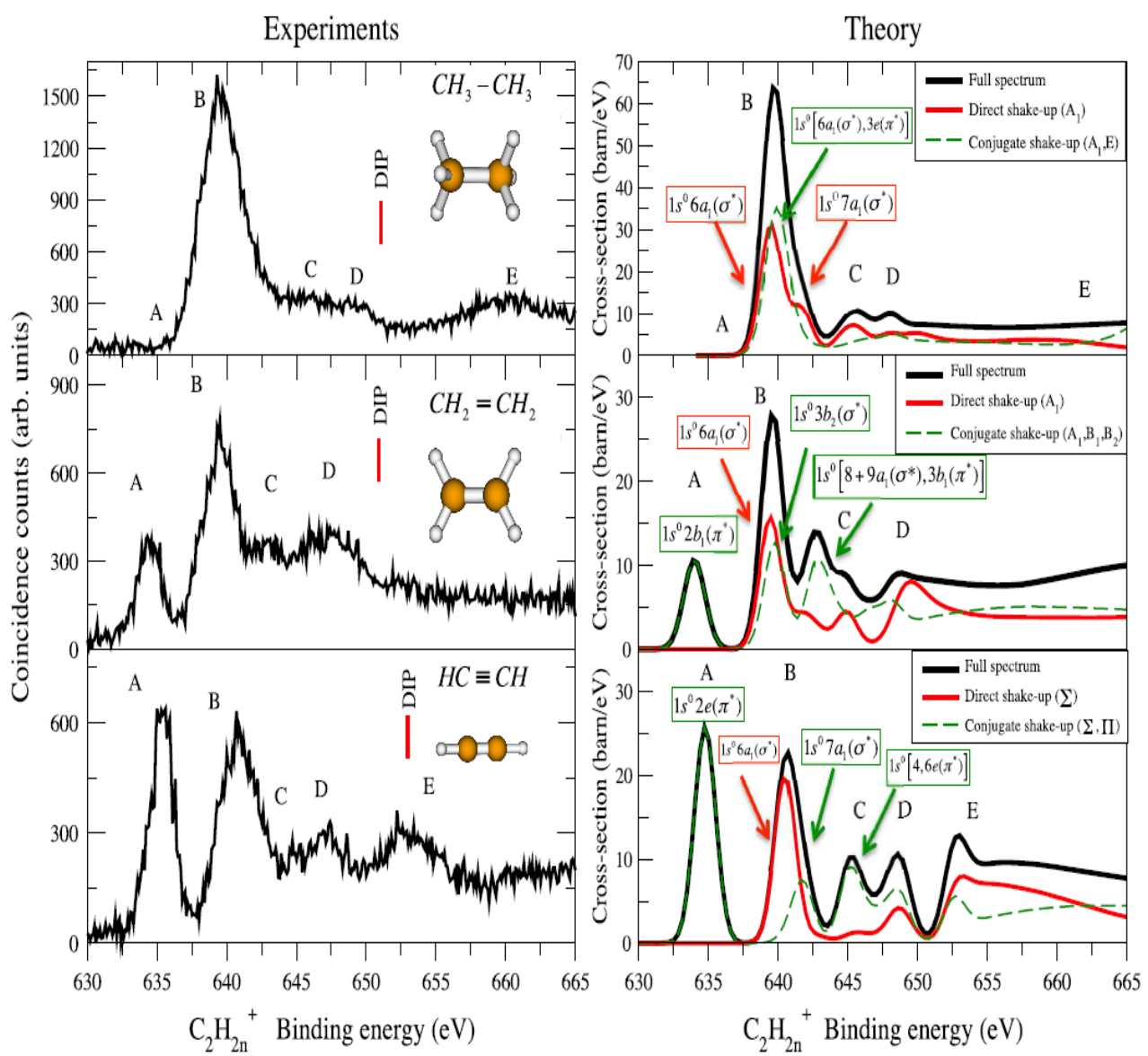

Fig. 14: Left: Experimental $\mathrm{K}^{-2} \mathrm{~V}$ states in $\mathrm{C}_{2} \mathrm{H}_{2 n}$ molecules recorded at a photon energy of $770 \mathrm{eV}$. The red line locates the $\mathrm{K}^{-2}$ threshold (or double ionization potential, DIP). Right: Absolute $\mathrm{K}^{-2} \mathrm{~V}$ cross sections. A convolution with a FWHM $1.8 \mathrm{eV}$ Gaussian was used to simulate experimental resolution. Dashed green, solid red, and thick black lines represent, respectively, the conjugate and the direct components and their incoherent sum. Assignments are given for the main contributions. Reprinted with permission from [61] 

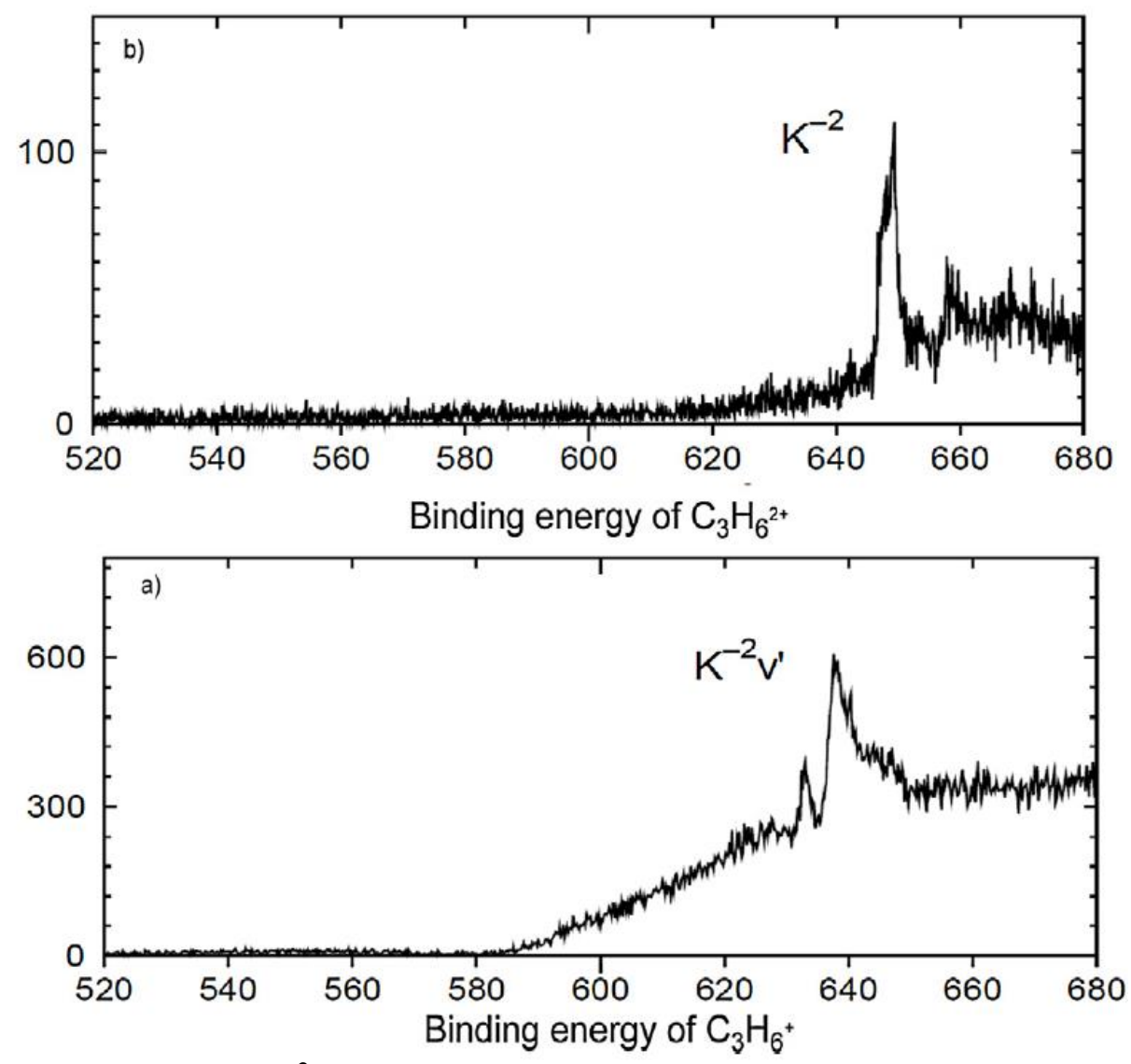

Fig. 15: (a) Propylene $\mathrm{K}^{-2} \mathrm{~V}$ spectrum obtained by detecting one photoelectron in coincidence with two Auger electrons at a photon energy of $710 \mathrm{eV}$. The background is due to core-valence double photoionization processes that have not been subtracted. The first peak at $635 \mathrm{eV}$ is due to $\pi^{*}$ resonance. (b) Associated $\mathrm{K}^{-2}$ spectrum showing the composite structure of the main peak due to the three different $\mathrm{C}$ atoms. Reprinted with permission from [9] 

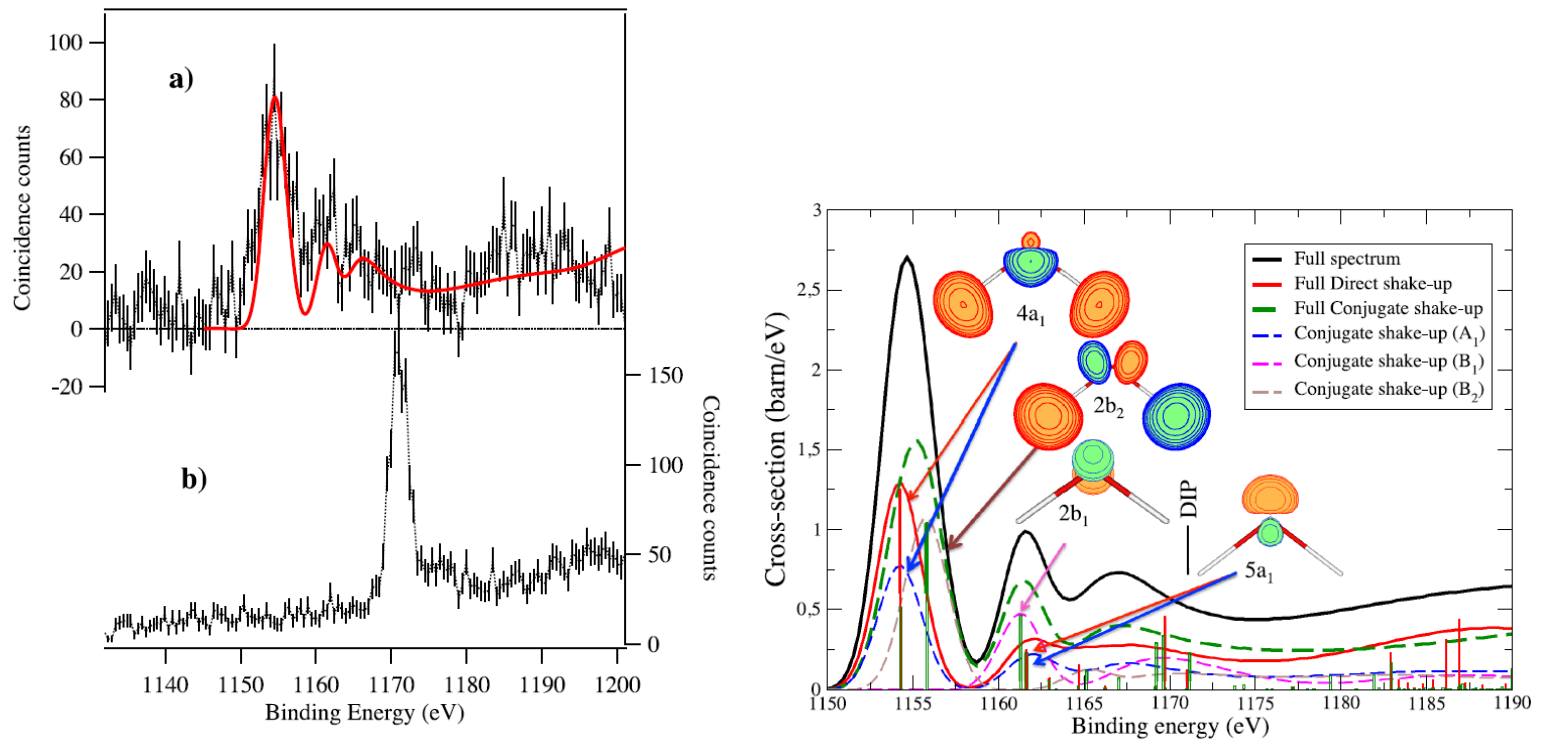

Fig. 16: $\mathrm{K}^{-2} \mathrm{~V}$ spectra in $\mathrm{H}_{2} \mathrm{O}$. Left = experiment at a photon energy of $1300 \mathrm{eV}$. a) and b) are respectively the $\mathrm{K}^{-2} \mathrm{~V}$ and the $\mathrm{K}^{-2}$ spectra. Red line is theory. Right gives the detailed $\mathrm{K}^{-2} \mathrm{~V}$ theoretical spectrum with the conjugate and direct contributions. Reprinted with permission from [65].
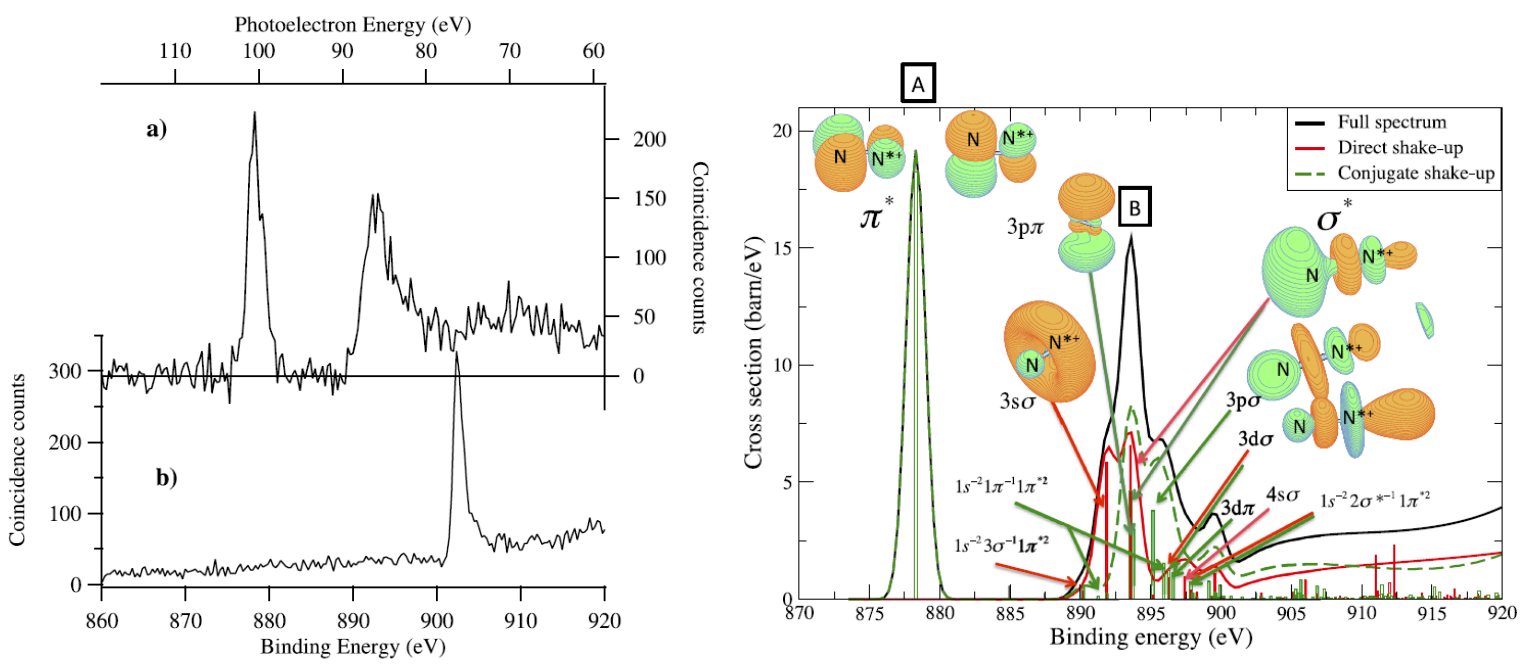

Fig. 17: $\mathrm{K}^{-2} \mathrm{~V}$ spectra in $\mathrm{N}_{2}$. Left = experiment at a photon energy of $979 \mathrm{eV}$. a) and b) are respectively the $\mathrm{K}^{-2} \mathrm{~V}$ and the $\mathrm{K}^{-2}$ spectra. Right gives the detailed $\mathrm{K}^{-2} \mathrm{~V}$ theoretical spectrum with the conjugate and direct contributions. Reprinted with permission from [68]. 


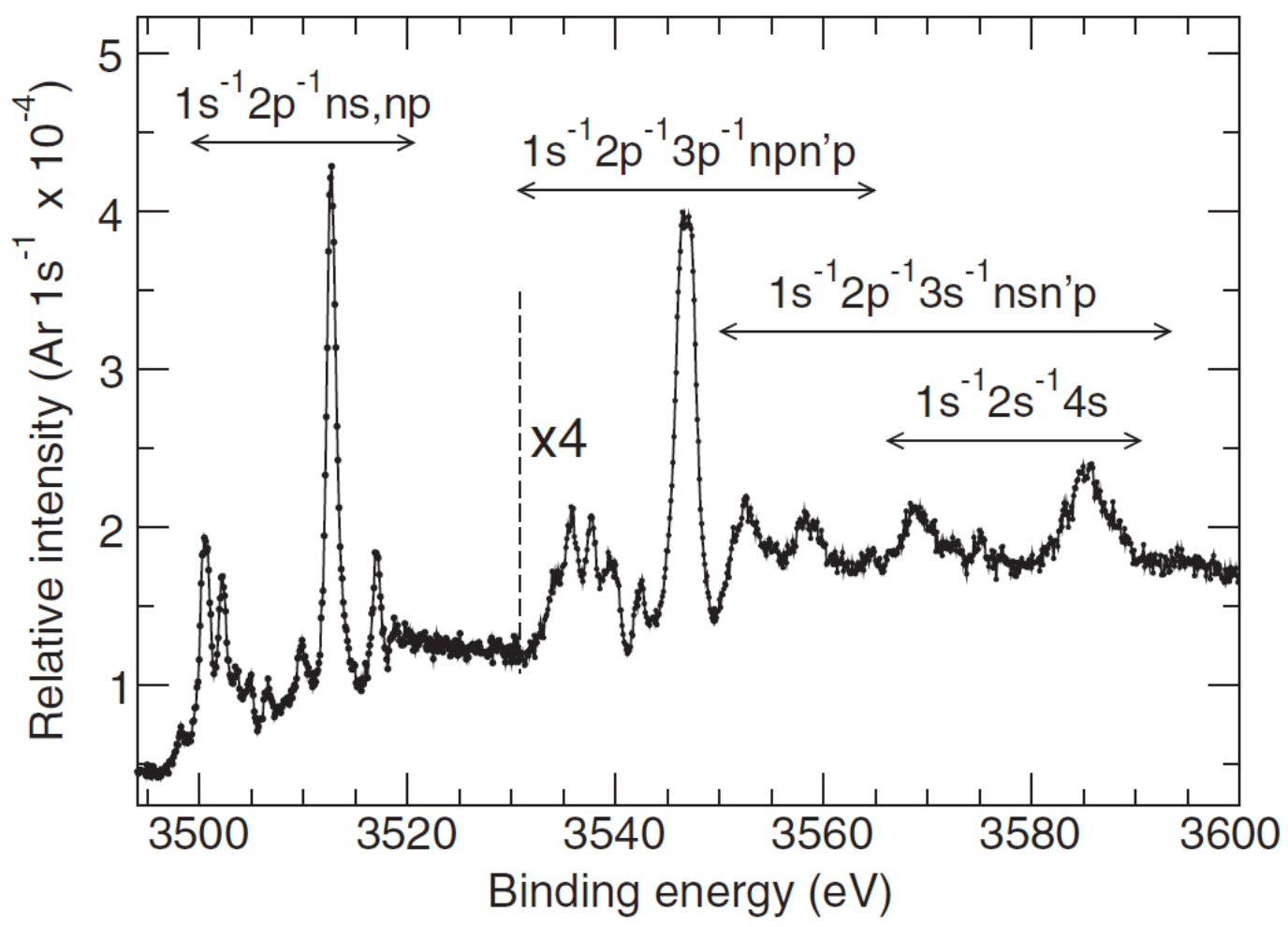

Fig. 18: The $\mathrm{K}^{-1} \mathrm{~L}^{-1} \mathrm{~V}$ spectrum of argon, relative to the intensity of $\mathrm{Ar} 1 \mathrm{~s}^{-1}$ photoionization. Above $3530 \mathrm{eV}$, the relative intensity is multiplied by a factor of 4 . The spectrum is obtained from the non-coincident detection of the photoelectron emitted upon $\mathrm{K}^{-1} \mathrm{~L}^{-1} \mathrm{~V}$ formation. Reprinted with permission from [70]. 